\title{
TRANSMISSION, REFLECTION AND SECOND-HARMONIC GENERATION IN A NONLINEAR WAVEGUIDE
}

\author{
ROBERTO CAMASSA ${ }^{1,2}$ \\ ALP FINDIKOGLU ${ }^{3}$ \\ GRANT LYTHE 4
}

\begin{abstract}
.
We present an experimental, analytical and numerical investigation of the passage of electromagnetic signals through a device with voltage-dependent differential capacitance. This dependence gives rise to the device's nonlinear response, which can then be tuned by an externally applied static electric field. The system is modeled with a wave equation for the current and the charge density with continuity conditions at the boundaries between two linear regions and the nonlinear medium they sandwich. We derive asymptotic formulae for transmission and reflection coefficients of a monochromatic signal and its nonlinearity-induced second harmonics. Predictions based on this analysis are then compared with numerical and experimental results, across a range of parameters values, including those tuning the nonlinearity by means of an imposed voltage. The experiments are carried out at microwave frequencies using $1 \mathrm{~cm}^{2}$ devices consisting of a superconducting thin film meandering waveguide on a nonlinear dielectric substrate.
\end{abstract}

Key words. Transmission and reflection, tunable nonlinearity, finite length nonlinear medium, second-harmonic, differential capacitance, boundaries, method of characteristics, noise, numerical solution.

AMS subject classifications. subject classifications: 35-04, 35L70, 78-05, 78M20.

1. Introduction. In this paper we study transmission, reflection and generation of harmonics when an electromagnetic signal passes through a finite-length region, which represents a nonlinear medium coupled via realistic boundary conditions to its environment. Our theory is developed with actual experimental devices in mind, but is applicable to any system where waves pass through a medium with voltagedependent differential capacitance, i.e. the charge does not simply increase linearly with voltage [1], and where losses are not so strong as to overwhelm nonlinear effects. Of particular interest in this class of media is the consequence that the functional form of nonlinearity is tunable: the device response can be controlled with an external bias voltage, which enables exploration of dynamical behaviors characteristic of quadratic and cubic nonlinear media within the same apparatus [2]-[11].

The experimental data we collect are obtained by sending electromagnetic signals along an $8 \mathrm{~cm}$ length meandering waveguide consisting of superconducting electrodes on the surface of a $10 \mathrm{~mm} \times 10 \mathrm{~mm} \times 0.5 \mathrm{~mm}$ nonlinear dielectric crystal of strontium titanate [10]-[15]. Electromagnetic waves in the waveguide with wavelengths of a few $\mathrm{cm}$ have frequencies of a few hundred $\mathrm{MHz}$ (microwaves). We model this experimental set-up with a wave equation coupling the evolution of voltage and current through a distributed capacitance that depends on voltage and temperature throughout the nonlinear region. Section 2 formulates this model in its nondimensional form used for analytical and numerical studies. After introducing the nonlinear wave equation and the definition of differential capacitance, we express it in terms of its characteristic variables and the corresponding Riemann invariants [16]. Because of the boundary conditions, this form cannot be immediately used to provide closed form solutions.

\footnotetext{
${ }^{1}$ Department of Mathematics, University of North Carolina at Chapel Hill, NC 27599, USA.

3 Superconductivity Technology Center, Los Alamos National Laboratory, Los Alamos, New Mexico 87545, USA.

${ }^{4}$ Department of Applied Mathematics, University of Leeds, Leeds LS2 9JT, UK.
} 
We then proceed by carrying out an asymptotic expansion in these variables in the limit of low amplitude incident signals, through a corresponding expansion of the boundary conditions. The analysis is simplified by the absence of dispersion and dissipation in the nonlinear wave equation, and produces closed form expressions for the nonlinear contributions to experimentally measurable quantities, such as transmission a reflection coefficients.

In Section 3, we study the concrete case of a sinusoidal input from the left of the region. In the weakly nonlinear regime, we carry out an asymptotic expansion of the characteristics solution in the parameter $a / v^{*}$, where $a$ is the amplitude of the input signal and $v^{*}$ is the characteristic voltage associated with the variation of the differential capacitance in the nonlinear central region. The calculation of the secondharmonic generated signal is given in some detail, in Section 3 and Appendix A, since this establishes a basis for further research on this class of system. The analysis yields explicit formulae that characterize the frequency-doubling response caused by nonlinearity.

Section 4 reports on numerical simulations of the governing equations: two coupled PDEs for the charge and current fields in three regions. The charge and current fields are updated in such a way as to keep current and voltage continuous across the boundaries, even though the charge-voltage relationship has a discontinuity. Sinusoidal signals and noise, band-limited or white, are continuously input from the left. Fourier transforms of numerical time series in the steady state, at points in the left and right regions, are used to evaluate the transmitted and reflected signals. We find good quantitative agreement with analytical results. In particular, we are able to predict the amplitude of the second-harmonic generated by the nonlinearity.

In Section 5 we compare the analytical and numerical results with experimental data. These are collected for microwave signals and noise passing through a compact device that operates at temperatures that are easily attained using liquid nitrogen or helium, and whose response can be tuned with a bias voltage of a few Volts. The waveguide is a patterned superconducting thin film; because its lateral dimensions are much smaller than the wavelengths of the input signals, wave propagation is effectively one-dimensional. The source of the nonlinearity is the nonlinear dielectric substrate that the superconducting waveguide rests on[19, 20, 21]. We find very good agreement with all the major measurable quantities of interest, while qualitative agreement is achieved when effects neglected by our model, but known to become relevant in certain regimes (like high frequencies), come into play.

2. The governing equations. The dynamics of wave propagation along the transmission line is described by the wave equation for the current $i(x, t)$ (Coulomb $\mathrm{s}^{-1}$ ) and the charge density $q(x, t)\left(\right.$ Coulomb $\left.\mathrm{m}^{-1}\right)$

$$
\begin{aligned}
\frac{\partial q(x, t)}{\partial t} & =-\frac{\partial i(x, t)}{\partial x} \\
L \frac{\partial i(x, t)}{\partial t} & =-\frac{\partial v(x, t)}{\partial x}
\end{aligned}
$$

where $L$ is the inductance per unit length. A relationship between $q(x, t)$ and the voltage $v(x, t)$ is needed to close the set of equations (2.1). Let

$$
q(x, t)=\mathcal{Q}(v(x, t))
$$


The "differential capacitance" [1] is the derivative of $\mathcal{Q}(v)$ :

$$
C_{\mathrm{d}}(v)=\frac{\mathrm{d} \mathcal{Q}(v)}{\mathrm{d} v} .
$$

In a linear medium $q(x, t)=C v(x, t)$, where $C$ is constant. Then the differential capacitance is the constant $C$ and the wave equation (2.1) is linear:

$$
\frac{\partial^{2}}{\partial t^{2}} v(x, t)=(L C)^{-1} \frac{\partial^{2}}{\partial x^{2}} v(x, t) .
$$

The nonlinear wave equation we study in this work can be written as

$$
L C_{\mathrm{d}}(v(x, t)) \frac{\partial^{2}}{\partial t^{2}} v(x, t)+L \frac{\partial C_{\mathrm{d}}(v(x, t)}{\partial v} \frac{\partial v(x, t)}{\partial t}=\frac{\partial^{2}}{\partial x^{2}} v(x, t) .
$$

Motivated by the configuration of the experiments, and by analogy with classical transmission-reflection problems, we study the propagation of waves through a nonlinear region (region II, $0 \leq x \leq l$ ) sandwiched between two semi-infinite regions (region I, $-\infty<x<0$ and region III, $l<x<\infty$ ) where the wave equation is linear. Current and voltage are continuous at the boundaries. The situation is illustrated in Figure 2.1.

We shall assume that the relationship (2.2) is such that we can define its inverse: $v(x, t)=\mathcal{Q}^{-1}(q(x, t))$. The inductance and differential capacitance per unit length are as follows:

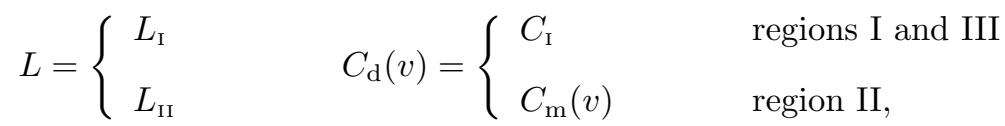

where $L_{\mathrm{I}}, L_{\mathrm{II}}$ and $C_{\mathrm{I}}$ are constants. We assume that $C_{\mathrm{m}}(v)$ is a positive even function with maximum at $v=0$. See Figure 2.2. Since $C_{\mathrm{m}}^{\prime}(0)=0$ and $C_{\mathrm{m}}^{\prime \prime}(0)<0$ we define the characteristic voltage associated with the differential capacitance curve by

$$
v^{*}=\left(\frac{2 C_{\mathrm{m}}(0)}{\left|C_{\mathrm{m}}^{\prime \prime}(0)\right|}\right)^{\frac{1}{2}} .
$$

A constant "bias" voltage $v_{\mathrm{b}}$ is applied across the three regions. The voltage at time $t$ and position $x$ is thus the sum of $v_{\mathrm{b}}$ and the time-dependent voltages due to the input signals and their interactions. The input signals consist of one or more sinusoidal signals and broadband noise. Noise effects are interesting in their own right [11], but in this paper we shall use noise input as a technique to explore simultaneously the response at numerous frequencies.

In Section 3 we consider the case where the input signal (the right-going wavetrain in region I) is given by $v_{\text {in }}=a \cos (2 \pi f(t-x / u))$. Our analysis is based on the assumption that $a / v^{*} \ll 1$. The bias voltage is not assumed small, but is fixed for any one experimental or numerical run. Thus the voltage values attained in any one experiment are in a small interval of the differential capacitance curve, but the full catalog of nonlinear behaviors can be explored by performing runs at various values of the bias voltage.

We define the dimensionless fields

$$
V(x, t)=\frac{v(x, t)}{v^{*}},
$$




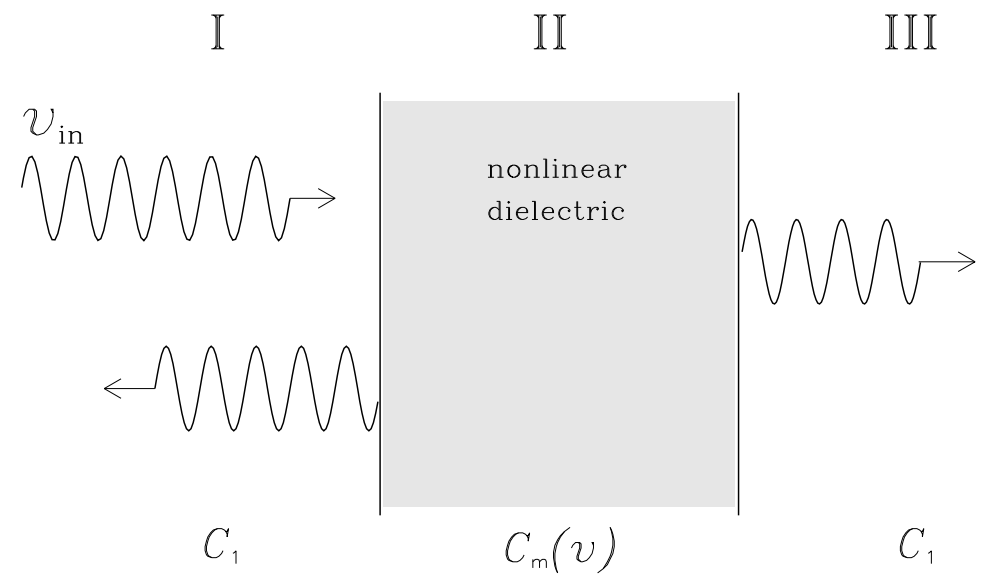

FIG. 2.1. Schematic diagram for solution of the nonlinear wave equation in three onedimensional regions. A constant bias voltage $v_{b}$ is applied across all three regions and the right-going wavetrain in region $I$ is the input signal, $v_{\mathrm{in}}$. The late-time solution has the form of an incident and reflected wavetrain in region I and a transmitted wavetrain in region III.

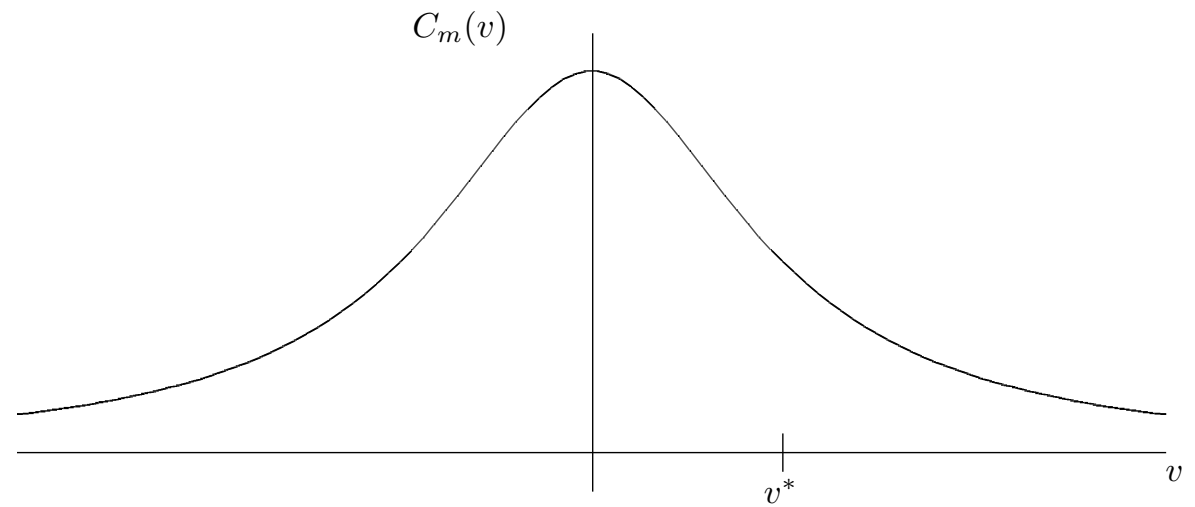

FIG. 2.2. A typical graph of differential capacitance versus voltage. The voltage $v^{*}$ is defined in (2.7)

$$
\begin{aligned}
Q(x, t) & =\frac{q(x, t)}{C_{\mathrm{II}} v^{*}}, \\
I(x, t) & =\left(\frac{L_{\mathrm{II}}}{C_{\mathrm{II}}}\right)^{\frac{1}{2}} \frac{i(x, t)}{v^{*}},
\end{aligned}
$$

where

$$
C_{\mathrm{II}}=C_{\mathrm{m}}(0)
$$

In these variables, $(2.1)$ is

$$
\frac{\partial}{\partial t}\left(\begin{array}{c}
Q(x, t) \\
I(x, t)
\end{array}\right)=-\left(L_{\mathrm{II}} C_{\mathrm{II}}\right)^{-\frac{1}{2}} \frac{\partial}{\partial x}\left(\begin{array}{c}
I(x, t) \\
V(x, t)
\end{array}\right),
$$


where $V(x, t)$ is given as a function of $Q(x, t)$ by

$$
V(x, t)=\left\{\begin{array}{lr}
\frac{L_{\mathrm{II}} C_{\mathrm{II}}}{L_{\mathrm{I}} C_{\mathrm{I}}} Q(x, t), & \text { regions I and III } \\
\frac{1}{v^{*}} \mathcal{Q}^{-1}\left(C_{\mathrm{II}} v^{*} Q(x, t)\right) . & \text { region II, }
\end{array}\right.
$$

We also nondimensionalize space and time by dividing by the length $l$ of the waveguide and the transit time at the velocity of light in a vacuum, $c$ :

$$
X=\frac{x}{l} \quad T=t \frac{c}{l} .
$$

So region $\mathrm{I}$ is $-\infty<X<0$, region II is $0 \leq X \leq 1$ and region III is $1<X<\infty$. Then (2.10) becomes

$$
\frac{\partial}{\partial T}\left(\begin{array}{c}
Q(X, T) \\
I(X, T)
\end{array}\right)=-U \frac{\partial}{\partial X}\left(\begin{array}{c}
I(X, T) \\
V(X, T)
\end{array}\right)
$$

where

$$
U=\frac{1}{c}\left(L_{\mathrm{II}} C_{\mathrm{II}}\right)^{-\frac{1}{2}} .
$$

The propagation speed of small-amplitude signals in region II, $u_{\mathrm{m}}\left(v_{\mathrm{b}}\right)$ is

$$
u_{\mathrm{m}}\left(v_{\mathrm{b}}\right)=\left(L_{\mathrm{II}} C_{\mathrm{m}}\left(v_{\mathrm{b}}\right)\right)^{-\frac{1}{2}} ;
$$

the constant $U=u_{\mathrm{m}}(0) / c$ is the dimensionless speed at zero bias. In our numerical work, we solve the nonlinear PDEs in the form (2.12).

Our analytical work, using the method of characteristics, proceeds by rewriting (2.12), using

$$
\frac{\mathrm{d} Q}{\mathrm{~d} T}=\frac{\mathrm{d} Q}{\mathrm{~d} V} \frac{\mathrm{d} V}{\mathrm{~d} T}
$$

in the form

$$
\frac{\partial}{\partial X}\left(\begin{array}{c}
I \\
V
\end{array}\right)=-U^{-1}\left(\begin{array}{cc}
0 & G(V) \\
1 & 0
\end{array}\right) \frac{\partial}{\partial T}\left(\begin{array}{c}
I \\
V
\end{array}\right)
$$

where the arguments $(X, T)$ for the fields $V$ and $I$ have been suppressed and

$$
G(V)=\left\{\begin{array}{lr}
G_{1} & \text { regions I and III } \\
G_{\mathrm{n}}(V) & \text { region II, }
\end{array}\right.
$$

where

$$
G_{\mathrm{l}}=\frac{L_{\mathrm{I}} C_{\mathrm{I}}}{L_{\mathrm{II}} C_{\mathrm{II}}},
$$

a constant, and

$$
G_{\mathrm{n}}(V)=\frac{C_{\mathrm{m}}\left(v^{*} V\right)}{C_{\mathrm{II}}} .
$$




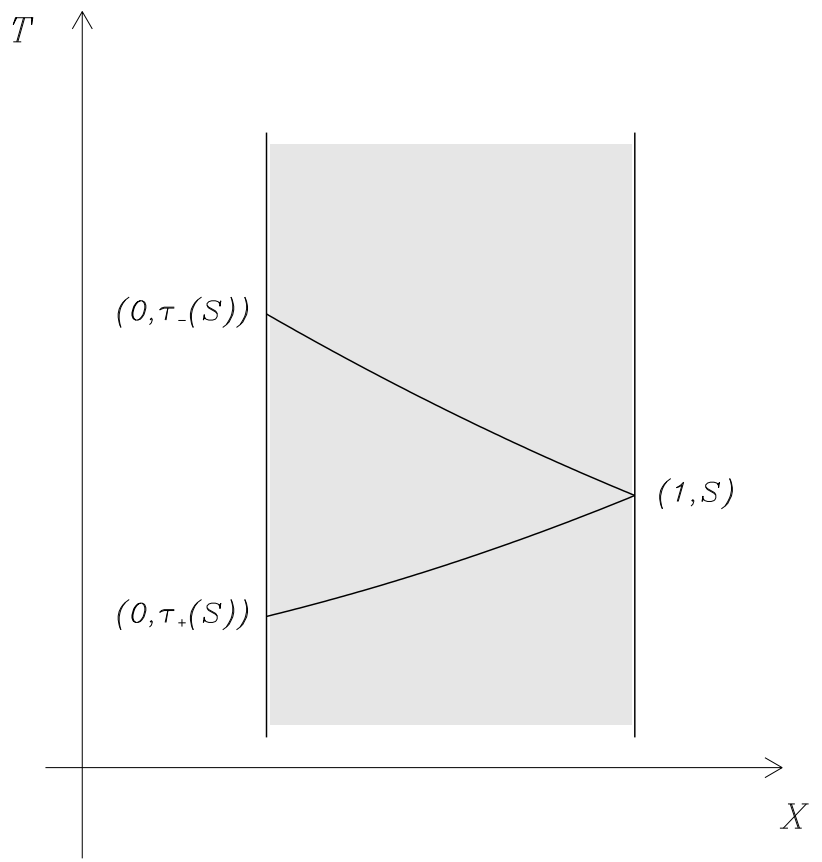

FIG. 2.3. The "initial times" functions. The two characteristic curves that cross at $(1, S)$ follow paths that intersect $X=0$ at times $\tau_{+}(S)$ and $\tau_{-}(S)$.

As $V \rightarrow 0, G_{\mathrm{n}}(V) \rightarrow 1-V^{2}+\mathcal{O}\left(V^{4}\right)$.

In order to use the method of characteristics [16] in Region II, we introduce the fields $\Gamma_{+}(X, T)$ and $\Gamma_{-}(X, T)$, defined as

$$
\Gamma_{ \pm}=H(V) \pm I
$$

where

$$
H^{\prime}(V)=\left(G_{\mathrm{n}}(V)\right)^{\frac{1}{2}}
$$

The fields $\Gamma_{ \pm}(X, T)$ are constants on two characteristic curves in the $(X, T)$ plane defined by the solutions $T_{ \pm}(X)$ of the equations

$$
\frac{\mathrm{d} T_{ \pm}}{\mathrm{d} X}= \pm U^{-1}\left(G_{\mathrm{n}}\left(V\left(X, T_{+}\right)\right)\right)^{\frac{1}{2}} .
$$

We shall impose conservation of the fields $\Gamma_{ \pm}(X, T)$ along characteristics using "initial times functions": given that a characteristic curve passes through $X=1$ at time $S$, the time at which it passes through $X=0$ is

$$
\tau_{ \pm}(S)=\left.T_{ \pm}(0)\right|_{T(1)=S} .
$$

Then

$$
\Gamma_{ \pm}\left(0, \tau_{ \pm}(S)\right)=\Gamma_{ \pm}(1, S) .
$$


See Figure 2.3. At the boundary between region I and region II, $X=0$, we denote

$$
\begin{aligned}
V(0, T) & =V_{\mathrm{I}}(T) \\
I(0, T) & =I_{\mathrm{I}}(T) .
\end{aligned}
$$

At the boundary between region II and region III, $X=1$, we denote

$$
\begin{aligned}
V(1, T) & =V_{\mathrm{III}}(T) \\
I(1, T) & =I_{\mathrm{III}}(T) .
\end{aligned}
$$

The conditions (2.23) can be rewritten

$$
H\left(V_{\mathrm{I}}\left(\tau_{ \pm}(S)\right)\right) \pm I_{\mathrm{I}}\left(\tau_{ \pm}(S)\right)=H\left(V_{\mathrm{III}}(S)\right) \pm I_{\mathrm{III}}(S) .
$$

In Section 3, we evaluate the transmitted signal as a function of the input signal using (2.26).

3. Transmission, reflection and harmonic generation: analytical results. Let the input signal be sinusoidal with amplitude $a$ at frequency $f$, that is

$$
v_{\text {in }}(x, t)=a \cos (2 \pi f(t-x / u)),
$$

where $u=\left(L_{\mathrm{I}} C_{\mathrm{I}}\right)^{-\frac{1}{2}}$. We nondimensionalize the bias voltage and input amplitude:

$$
A=\frac{a}{v^{*}} \quad \text { and } \quad V_{\mathrm{b}}=\frac{v_{\mathrm{b}}}{v^{*}},
$$

where $v^{*}$ is defined in (2.7). The solution of (2.16) can be expanded as

$$
V(X, T)=V_{\mathrm{b}}+A V^{(0)}(X, T)+A^{2} V^{(1)}(X, T)+\mathcal{O}\left(A^{3}\right) .
$$

Thus

$$
V_{\mathrm{I}}(T)=V(0, T)=V_{\mathrm{b}}+A V_{\mathrm{I}}^{(0)}(T)+A^{2} V_{\mathrm{I}}^{(1)}(T)+\mathcal{O}\left(A^{3}\right) ;
$$

$I_{\mathrm{I}}(T), V_{\mathrm{III}}(T)$ and $I_{\mathrm{III}}(T)$ will be written in a similar way.

The function $H(V(X, T))$ is expanded as

$$
\begin{aligned}
H(V(X, T))=H\left(V_{\mathrm{b}}\right)+ & H^{\prime}\left(V_{\mathrm{b}}\right)\left(V(X, T)-V_{\mathrm{b}}\right)+\frac{1}{2} H^{\prime \prime}\left(V_{\mathrm{b}}\right)\left(V(X, T)-V_{\mathrm{b}}\right)^{2}+\mathcal{O}\left(A^{3}\right) \\
=H\left(V_{\mathrm{b}}\right)+ & G_{\mathrm{n}}\left(V_{\mathrm{b}}\right)^{\frac{1}{2}}\left(A V^{(0)}(X, T)+A^{2} V^{(1)}(X, T)+\frac{1}{4} A^{2} \frac{G_{\mathrm{n}}^{\prime}\left(V_{\mathrm{b}}\right)}{G_{\mathrm{n}}\left(V_{\mathrm{b}}\right)}\left(V^{(0)}(X, T)\right)^{2}\right) \\
& +\mathcal{O}\left(A^{3}\right),
\end{aligned}
$$

and the characteristic curves and initial times functions as

$$
T_{ \pm}(X)=T_{ \pm}^{(0)}(X)+A T_{ \pm}^{(1)}(X)+\mathcal{O}\left(A^{2}\right),
$$

and

$$
\tau_{ \pm}(S)=\tau_{ \pm}^{(0)}(S)+A \tau_{ \pm}^{(1)}(S)+\mathcal{O}\left(A^{2}\right)
$$


Finally, the equality (2.26) is expanded as

$$
\begin{aligned}
& G_{\mathrm{n}}\left(V_{\mathrm{b}}\right)^{\frac{1}{2}}\left[A V_{\mathrm{I}}^{(0)}\left(\tau_{ \pm}^{(0)}(S)+A \tau_{ \pm}^{(1)}(S)\right)\right.\left.+A^{2}\left(V_{\mathrm{I}}^{(1)}\left(\tau_{ \pm}^{(0)}(S)\right)+\frac{1}{4} \frac{G_{\mathrm{n}}^{\prime}\left(V_{\mathrm{b}}\right)}{G_{\mathrm{n}}\left(V_{\mathrm{b}}\right)} V_{\mathrm{I}}^{(0)}\left(\tau_{ \pm}^{(0)}(S)\right)^{2}\right)\right] \\
& \pm {\left[A I_{\mathrm{I}}^{(0)}\left(\tau_{ \pm}^{(0)}(S)+A \tau_{ \pm}^{(1)}(S)\right)+A^{2} I_{\mathrm{I}}^{(1)}\left(\tau_{ \pm}^{(0)}(S)\right)\right] } \\
&=G_{\mathrm{n}}\left(V_{\mathrm{b}}\right)^{\frac{1}{2}}\left[A V_{\mathrm{III}}^{(0)}(S)+A^{2}\left(V_{\mathrm{III}}^{(1)}(S)+\frac{1}{4} \frac{G_{\mathrm{n}}^{\prime}\left(V_{\mathrm{b}}\right)}{G_{\mathrm{n}}\left(V_{\mathrm{b}}\right)} V_{\mathrm{III}}^{(0)}(S)^{2}\right)\right] \\
& \pm\left[A I_{\mathrm{III}}^{(0)}(S)+A^{2} I_{\mathrm{III}}^{(1)}(S)\right]+\mathcal{O}\left(A^{3}\right) .
\end{aligned}
$$

In Section 3.1, we shall impose (3.7) keeping only terms proportional to $A$. The result is the classical transmission-reflection relation for finite-length linear media: perfect transmission is found at frequencies such that the length of region II is a multiple of half the wavelength of the input sinusoid. In Section 3.2, we shall also keep terms proportional to $A^{2}$ in (3.7).

3.1. Lowest order. To order $A$, the characteristic curves (2.21) and (2.21) are straight lines:

$$
T_{ \pm}^{(0)}(X)=T_{ \pm}^{(0)}(0) \pm \frac{X}{U_{\mathrm{m}}}
$$

where the normalized speed of propagation in region II is

$$
U_{\mathrm{m}}=G_{\mathrm{n}}\left(V_{\mathrm{b}}\right)^{-\frac{1}{2}} U=\frac{1}{c}\left(L_{\mathrm{II}} C_{\mathrm{m}}\left(V_{\mathrm{b}}\right)\right)^{-\frac{1}{2}} .
$$

Thus the initial times functions at order $A$ are simply

$$
\tau_{ \pm}^{(0)}(S)=S \mp U_{\mathrm{m}}^{-1} \text {. }
$$

To order $A$, the invariant functions along the characteristics are

$$
\Gamma_{ \pm}^{(0)}(X, T)=G_{\mathrm{n}}\left(V_{\mathrm{b}}\right)^{\frac{1}{2}} V^{(0)}(X, T) \pm I^{(0)}(X, T)
$$

and the condition $(2.26)$ is

$$
G_{\mathrm{n}}\left(V_{\mathrm{b}}\right)^{\frac{1}{2}} V_{\mathrm{I}}^{(0)}\left(S \mp U_{\mathrm{m}}^{-1}\right) \pm I_{\mathrm{I}}^{(0)}\left(S \mp U_{\mathrm{m}}^{-1}\right)=G_{\mathrm{n}}\left(V_{\mathrm{b}}\right)^{\frac{1}{2}} V_{\mathrm{III}}^{(0)}(S) \pm I_{\mathrm{III}}^{(0)}(S)
$$

Notice that (3.8) and (3.12) apply to the following linear wave equation:

$$
\frac{\partial}{\partial X}\left(\begin{array}{c}
I^{(0)} \\
V^{(0)}
\end{array}\right)=-U^{-1}\left(\begin{array}{cc}
0 & G\left(V_{\mathrm{b}}\right) \\
1 & 0
\end{array}\right) \frac{\partial}{\partial T}\left(\begin{array}{c}
I^{(0)} \\
V^{(0)}
\end{array}\right) .
$$

The function $G\left(V_{\mathrm{b}}\right)$ is piecewise constant, so the speed of propagation in region II depends on bias voltage.

With a continuous sinusoidal input, (3.1), the solution of (3.13) after a transient has the form of an incident and reflected wavetrain in region $I$ and a transmitted wavetrain in region III. In region I, as $T \rightarrow \infty$, let

$$
\begin{aligned}
& V^{(0)}(X, T)=\frac{1}{2}\left(\exp \left(\mathrm{i} \Omega\left(T-\frac{X}{U_{\mathrm{I}}}\right)\right)+\mathcal{R}^{(0)}(\Omega) \exp \left(\mathrm{i} \Omega\left(T+\frac{X}{U_{\mathrm{I}}}\right)\right)+\text { c.c. }\right), \\
& (3.14) \quad \text { and } \\
& I^{(0)}(X, T)=\left(\frac{L_{\mathrm{II}} C_{\mathrm{I}}}{L_{\mathrm{I}} C_{\mathrm{II}}}\right)^{\frac{1}{2}} \frac{1}{2}\left(\exp \left(\mathrm{i} \Omega\left(T-\frac{X}{U_{\mathrm{I}}}\right)\right)-\mathcal{R}^{(0)}(\Omega) \exp \left(\mathrm{i} \Omega\left(T+\frac{X}{U_{\mathrm{I}}}\right)\right)+\text { c.c. }\right),
\end{aligned}
$$


where c.c. indicates complex conjugate and we have used the freedom to set the origin of $T$. The normalized speed of propagation in region $\mathrm{I}$ is

$$
U_{\mathrm{I}}=\frac{1}{c}\left(L_{\mathrm{I}} C_{\mathrm{I}}\right)^{-\frac{1}{2}},
$$

and the normalized signal frequency is

$$
\Omega=2 \pi f \frac{l}{c} .
$$

Similarly, in region III, let

$$
\begin{aligned}
& V^{(0)}(X, T)=\frac{1}{2}\left(\mathcal{T}^{(0)}(\Omega) \exp \left(\mathrm{i} \Omega\left(T-\frac{(X-1)}{U_{\mathrm{I}}}\right)\right)+\text { c.c. }\right) \\
& \text { and } \\
& I^{(0)}(X, T)=\left(\frac{L_{\mathrm{II}} C_{\mathrm{I}}}{L_{\mathrm{I}} C_{\mathrm{II}}}\right)^{\frac{1}{2}} \frac{1}{2}\left(\mathcal{T}^{(0)}(\Omega) \exp \left(\mathrm{i} \Omega\left(T-\frac{(X-1)}{U_{\mathrm{I}}}\right)\right)+\text { c.c. }\right) .
\end{aligned}
$$

Notice that the phase at $X=1$ is absorbed into $\mathcal{T}^{(0)}(\Omega)$.

The lowest-order transmission and reflection coefficients $\mathcal{T}^{(0)}(\Omega)$ and $\mathcal{R}^{(0)}(\Omega)$ are found by imposing the condition (3.12). Using (3.10), (3.14) and (3.17) gives explicit expressions for the functions $V_{\mathrm{I}}^{(0)}(T), I_{\mathrm{I}}^{(0)}(T), V_{\mathrm{III}}^{(0)}(T)$ and $I_{\mathrm{III}}^{(0)}(T)$ introduced in $(2.24)$ and $(2.25)$. Thus (3.12) is

$$
1+\mathcal{R}^{(0)}(\Omega) \pm \beta^{\frac{1}{2}}\left(1-\mathcal{R}^{(0)}(\Omega)\right)=\mathrm{e}^{ \pm \mathrm{i} \Omega U_{\mathrm{m}}^{-1}} \mathcal{T}^{(0)}(\Omega)\left(1 \pm \beta^{\frac{1}{2}}\right),
$$

where

$$
\beta=\frac{L_{\mathrm{II}} C_{\mathrm{I}}}{L_{\mathrm{I}} C_{\mathrm{m}}\left(v_{\mathrm{b}}\right)}=\frac{L_{\mathrm{II}} C_{\mathrm{I}}}{L_{\mathrm{I}} C_{\mathrm{II}}} \frac{1}{G_{\mathrm{n}}\left(V_{\mathrm{b}}\right)} .
$$

In the experimental system $\beta \ll 1$ because the impedance in Region II is higher than that in Regions I and III.

The pair of equations (3.18) can be solved to yield

$$
\begin{aligned}
\mathcal{R}^{(0)}(\Omega) & =-\frac{(1-\beta) \sin \left(\frac{\Omega}{U_{\mathrm{m}}}\right)}{(1+\beta) \sin \left(\frac{\Omega}{U_{\mathrm{m}}}\right)-2 \mathrm{i} \beta^{\frac{1}{2}} \cos \left(\frac{\Omega}{U_{\mathrm{m}}}\right)} \\
& =-\frac{\left(1-\beta^{2}\right) \sin ^{2}\left(\frac{\Omega}{U_{\mathrm{m}}}\right)-2 \mathrm{i} \beta^{\frac{1}{2}}(1-\beta) \sin \left(\frac{\Omega}{U_{\mathrm{m}}}\right) \cos \left(\frac{\Omega}{U_{\mathrm{m}}}\right)}{(1+\beta)^{2} \sin ^{2}\left(\frac{\Omega}{U_{\mathrm{m}}}\right)+4 \beta \cos ^{2}\left(\frac{\Omega}{U_{\mathrm{m}}}\right)}, \\
\mathcal{T}^{(0)}(\Omega) & =-\frac{2 \mathrm{i} \beta^{\frac{1}{2}}}{(1+\beta) \sin \left(\frac{\Omega}{U_{\mathrm{m}}}\right)-2 \mathrm{i} \beta^{\frac{1}{2}} \cos \left(\frac{\Omega}{U_{\mathrm{m}}}\right)} \\
& =-\frac{4 \beta \cos \left(\frac{\Omega}{U_{\mathrm{m}}}\right)+2 \mathrm{i} \beta^{\frac{1}{2}}(1+\beta) \sin \left(\frac{\Omega}{U_{\mathrm{m}}}\right)}{(1+\beta)^{2} \sin ^{2}\left(\frac{\Omega}{U_{\mathrm{m}}}\right)+4 \beta \cos ^{2}\left(\frac{\Omega}{U_{\mathrm{m}}}\right)} .
\end{aligned}
$$

These coefficients, also found in the quantum-mechanical problem of a potential step [17], satisfy the energy constraint $\left|\mathcal{R}^{(0)}(\Omega)\right|^{2}+\left|\mathcal{T}^{(0)}(\Omega)\right|^{2}=1$. Perfect transmission, i.e. $\left|\mathcal{T}^{(0)}(\Omega)\right|=1$, is found at resonant frequencies $\Omega$ satisfying

$$
\sin \left(\frac{\Omega}{U_{\mathrm{m}}}\right)=0
$$


3.2. Next order. In order to impose the equality (3.7) to order $A^{2}$ we write

$$
\begin{aligned}
& A V_{\mathrm{I}}^{(0)}\left(\tau_{ \pm}^{(0)}(S)+A \tau_{ \pm}^{(1)}(S)\right) \\
= & A V_{\mathrm{I}}^{(0)}\left(S \pm U_{\mathrm{m}}^{-1}\right)+A^{2} V_{\mathrm{I}}^{\prime}\left(S \pm U_{\mathrm{m}}^{-1}\right) \tau_{ \pm}^{(1)}(S)+\mathcal{O}\left(A^{3}\right),
\end{aligned}
$$

where $V_{\mathrm{I}}^{\prime}(T)=\frac{\mathrm{d}}{\mathrm{d} T} V_{\mathrm{I}}^{(0)}(T)$. The current $I_{\mathrm{I}}^{(0)}\left(\tau_{ \pm}^{(0)}(S)+A \tau_{ \pm}^{(1)}(S)\right)$ is expanded in the same way. To find the solution at order $A^{2}$, we therefore need an explicit form for the initial times function to order $A$. The relationship that gives the initial times for the two characteristics that cross at $(1, S)$ is

$$
\begin{aligned}
S= & \tau_{ \pm}(S) \pm \frac{1}{U} \int_{0}^{1}\left(G_{\mathrm{n}}\left(V\left(X, T_{ \pm}(X)\right)\right)^{\frac{1}{2}} \mathrm{~d} X\right. \\
= & \tau_{ \pm}^{(0)}(S)+A \tau_{ \pm}^{(1)}(S) \\
& \pm \frac{1}{U_{\mathrm{m}}} \int_{0}^{1}\left(1+\frac{1}{2} \frac{G_{\mathrm{n}}^{\prime}\left(V_{\mathrm{b}}\right)}{G_{\mathrm{n}}\left(V_{\mathrm{b}}\right)} A V^{(0)}\left(X, T_{ \pm}^{(0)}(X)\right)\right) \mathrm{d} X+\ldots
\end{aligned}
$$

Using (3.10) yields

$$
\tau_{ \pm}^{(1)}(S)=\mp \frac{1}{2} U_{\mathrm{m}}^{-1} \frac{G_{\mathrm{n}}^{\prime}\left(V_{\mathrm{b}}\right)}{G_{\mathrm{n}}\left(V_{\mathrm{b}}\right)} \int_{0}^{1} V^{(0)}\left(X, T_{ \pm}^{(0)}(X)\right) \mathrm{d} X .
$$

In Appendix A, we construct the explicit form of $V^{(0)}\left(X, T_{ \pm}^{(0)}\right)$ and perform the integral in (3.25), leading to the expansion (A.8) of (3.7) to order $A^{2}$.

The only sinusoidal terms on the right-hand side of (A.8) have frequency $2 \Omega$, so there is a transmitted second-harmonic signal with amplitude proportional to the square of the input amplitude [11]. We therefore let the non-constant terms of order $A^{2}$ in the expansion (3.4) be

$$
\begin{array}{lll}
V_{\mathrm{I}}^{(1)}(T)=\frac{1}{2}\left[\mathcal{R}^{(1)}(\Omega) \mathrm{e}^{2 \mathrm{i} \Omega T}+\text { c.c. }\right], & I_{\mathrm{I}}^{(1)}(T)=-\left(\frac{L_{\mathrm{II}} C_{\mathrm{I}}}{L_{\mathrm{I}} C_{\mathrm{II}}}\right)^{\frac{1}{2}} \frac{1}{2}\left[\mathcal{R}^{(1)}(\Omega) \mathrm{e}^{2 \mathrm{i} \Omega T}+\text { c.c. }\right] \\
V_{\mathrm{III}}^{(1)}(T)=\frac{1}{2}\left[\mathcal{T}^{(1)}(\Omega) \mathrm{e}^{2 \mathrm{i} \Omega T}+\text { c.c. }\right], & I_{\mathrm{III}}^{(1)}(T)=\left(\frac{L_{\mathrm{II}} C_{\mathrm{I}}}{L_{\mathrm{I}} C_{\mathrm{II}}}\right)^{\frac{1}{2}} \frac{1}{2}\left[\mathcal{T}^{(1)}(\Omega) \mathrm{e}^{2 \mathrm{i} \Omega T}+\text { c.c. }\right] .
\end{array}
$$

Then (A.8) becomes

$$
\begin{aligned}
& \left(1 \pm \beta^{\frac{1}{2}}\right) \mathcal{T}^{(1)}(\Omega)-\left(1 \mp \beta^{\frac{1}{2}}\right) \mathcal{R}^{(1)}(\Omega) \mathrm{e}^{\mp 2 \mathrm{i} \Omega U_{\mathrm{m}}^{-1}} \\
= & \frac{1}{8} \frac{G_{\mathrm{n}}^{\prime}\left(V_{\mathrm{b}}\right)}{G_{\mathrm{n}}\left(V_{\mathrm{b}}\right)} \mathcal{T}^{(0)}(\Omega)^{2}\left[\left(\cos \frac{\Omega}{U_{\mathrm{m}}}-\mathrm{i} \beta^{\frac{1}{2}} \sin \frac{\Omega}{U_{\mathrm{m}}}\right)^{2} \mathrm{e}^{\mp 2 \mathrm{i} \Omega U_{\mathrm{m}}^{-1}}-1\right. \\
& \left.\quad \mp \mathrm{i}\left(\left(1 \pm \beta^{\frac{1}{2}}\right)^{2} \frac{\Omega}{U_{\mathrm{m}}}+(1-\beta) \frac{1}{2}\left(\sin \left(2 \frac{\Omega}{U_{\mathrm{m}}}\right) \pm \mathrm{i}\left(1-\cos \left(2 \frac{\Omega}{U_{\mathrm{m}}}\right)\right)\right)\right)\right] .
\end{aligned}
$$

Explicit expressions for $\mathcal{T}^{(1)}(\Omega)$ and $\mathcal{R}^{(1)}(\Omega)$ can now be obtained by inverting the pair of complex equations (3.26). The result simplifies considerably at resonant frequencies, (3.22), when (3.26) reduces to

$$
\left(1 \pm \beta^{\frac{1}{2}}\right) \mathcal{T}^{(1)}(\Omega)-\left(1 \mp \beta^{\frac{1}{2}}\right) \mathcal{R}^{(1)}(\Omega)=\mp \mathrm{i}\left(1 \pm \beta^{\frac{1}{2}}\right)^{2} \frac{1}{8} \frac{G_{\mathrm{n}}^{\prime}\left(V_{\mathrm{b}}\right)}{G_{\mathrm{n}}\left(V_{\mathrm{b}}\right)} \frac{\Omega}{U_{\mathrm{m}}} .
$$


The solution of $(3.27)$ is

$$
\begin{aligned}
& \mathcal{T}^{(1)}(\Omega)=-\frac{1}{16} \frac{\mathrm{i}}{\beta^{\frac{1}{2}}} \frac{G_{\mathrm{n}}^{\prime}\left(V_{\mathrm{b}}\right)}{G_{\mathrm{n}}\left(V_{\mathrm{b}}\right)} \frac{\Omega}{U_{\mathrm{m}}}(1+3 \beta), \\
& \mathcal{R}^{(1)}(\Omega)=-\frac{1}{16} \frac{\mathrm{i}}{\beta^{\frac{1}{2}}} \frac{G_{\mathrm{n}}^{\prime}\left(V_{\mathrm{b}}\right)}{G_{\mathrm{n}}\left(V_{\mathrm{b}}\right)} \frac{\Omega}{U_{\mathrm{m}}}(1-\beta) .
\end{aligned}
$$

Note that $G^{\prime}(0)=0$ and $G^{\prime}(V)<0$ when $V \neq 0$. A relatively simple form is also found in the limit $\frac{\Omega}{U_{\mathrm{m}}} \gg 1$, when

$$
\begin{aligned}
\left(1 \pm \beta^{\frac{1}{2}}\right) \mathcal{T}^{(1)}(\Omega)-\left(1 \mp \beta^{\frac{1}{2}}\right) \mathcal{R}^{(1)}(\Omega) \mathrm{e}^{\mp 2 \mathrm{i} \Omega U_{\mathrm{m}}^{-1}} \\
\\
=\mp \mathcal{T}^{(0)}(\Omega)^{2} \mathrm{i}\left(1 \pm \beta^{\frac{1}{2}}\right)^{2} \frac{1}{8} \frac{G_{\mathrm{n}}^{\prime}\left(V_{\mathrm{b}}\right)}{G_{\mathrm{n}}\left(V_{\mathrm{b}}\right)} \frac{\Omega}{U_{\mathrm{m}}} .
\end{aligned}
$$

The solution of the system (3.30) is

$$
\begin{aligned}
& \mathcal{T}^{(1)}(\Omega)=-\frac{\mathrm{i}}{8} \mathcal{T}^{(0)}(\Omega)^{2} \frac{G_{\mathrm{n}}^{\prime}\left(V_{\mathrm{b}}\right)}{G_{\mathrm{n}}\left(V_{\mathrm{b}}\right)} \frac{\Omega}{U_{\mathrm{m}}} \frac{(1+3 \beta) \cos 2 \frac{\Omega}{U_{\mathrm{m}}}+\mathrm{i}\left(3 \beta^{\frac{1}{2}}+\beta^{\frac{3}{2}}\right) \sin 2 \frac{\Omega}{U_{\mathrm{m}}}}{2 \beta^{\frac{1}{2}} \cos 2 \frac{\Omega}{U_{\mathrm{m}}}+\mathrm{i}(1+\beta) \sin 2 \frac{\Omega}{U_{\mathrm{m}}}}, \\
& \mathcal{R}^{(1)}(\Omega)=-\frac{\mathrm{i}}{8} \mathcal{T}^{(0)}(\Omega)^{2} \frac{G_{\mathrm{n}}^{\prime}\left(V_{\mathrm{b}}\right)}{G_{\mathrm{n}}\left(V_{\mathrm{b}}\right)} \frac{\Omega}{U_{\mathrm{m}}} \frac{1-\beta}{2 \beta^{\frac{1}{2}} \cos 2 \frac{\Omega}{U_{\mathrm{m}}}+\mathrm{i}(1+\beta) \sin 2 \frac{\Omega}{U_{\mathrm{m}}}} .
\end{aligned}
$$

At the $n$th resonance, $\frac{\Omega}{U_{\mathrm{m}}}=n \pi$, so (3.31) is a rather good approximation in typical experimental situations where $n \geq 4$.

The factors on the right-hand-side of (3.31) have simple interpretations. The factor $\mathcal{T}^{(0)}(\Omega)^{2}$ means that the amplitude of the second harmonic is maximized when the frequency of the input signal is at resonance. The factor $G_{\mathrm{n}}^{\prime}\left(V_{\mathrm{b}}\right) / G_{\mathrm{n}}\left(V_{\mathrm{b}}\right)$ gives the explicit dependence on bias voltage; because $G_{\mathrm{n}}^{\prime}(0)=0$ there is no second harmonic generation at order $A^{2}$ for zero bias. The factor $\Omega=2 \pi f l / c$ means that the second harmonic amplitude is proportional to the "effective length" of the waveguide: the number of wavelengths over which the nonlinearity has time to act. The final factor involving transcendental functions is complicated. However it can be seen that the dependence on $2 \Omega / U_{\mathrm{m}}$ will produce secondary maxima in the second-harmonic amplitude at frequencies halfway between resonances of the input signal. Finally, we remark that the constant terms (zero-frequency "DC-component") of amplitude $A^{2}$, also generated by the quadratic component of nonlinearity, can always be absorbed into the bias voltage and hence their effects are already accounted for by $V_{b}$-variations.

4. Comparison with numerical solutions. The response of a finite-length nonlinear device was modeled by dividing the space into three one-dimensional regions. In the central region (region II) the wave equation, two coupled PDEs for the charge and current fields (2.12), is nonlinear. The numerical runs described in this Section used values of the capacitances and inductances, length of region II and input frequencies, chosen to be similar to those found in the experiments described in Section 5 below [23].

In the interior of each region, timestepping is performed according to the LaxWendroff method described in Appendix B.1. At the boundaries between region I and II and between regions II and III, we impose continuity of voltage and current. In Appendix B.2 we describe how this is made consistent with the different relations 


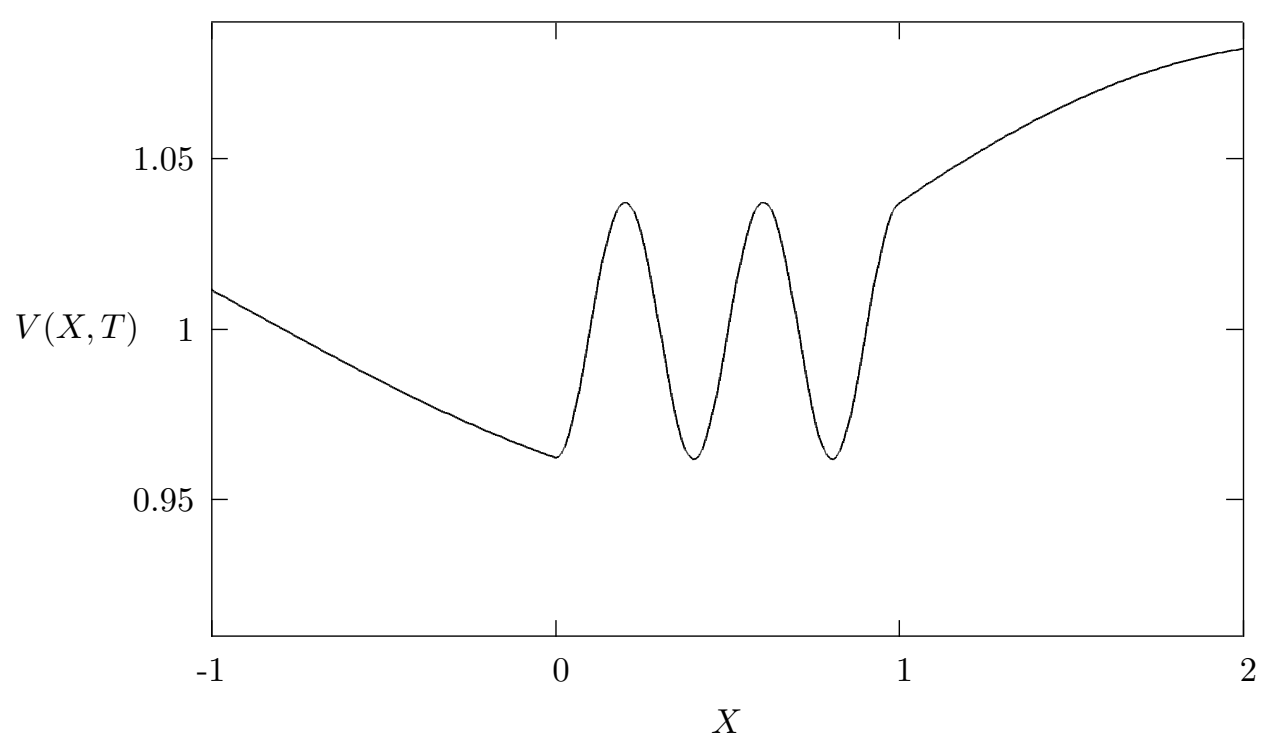

FIG. 4.1. Numerical voltage field as a function of $X$ for $T=1000$. In regions $I$ and III $(X<0$ and $X>1)$ the wave equation is linear; in region $I I(0 \leq X \leq 1)$, the wave equation is nonlinear. A sinusoidal signal was input at $f=250 \mathrm{MHz}$ with $a=0.1 \mathrm{~V}$. The parameters are $L_{\mathrm{I}} C_{\mathrm{I}} c^{2}=9$, $L_{\mathrm{II}} C_{\mathrm{II}} c^{2}=2500$ and $l=6 \mathrm{~cm}, v_{\mathrm{b}}=1 \mathrm{~V}$ and $v^{*}=10 \mathrm{~V}$. The partial differential equations (2.12) were solved with $\Delta X=0.001$ and $\Delta T=0.001$.

between charge and voltage functions that hold to the left and to the right of the boundary. In the numerics, we can examine the whole configuration of current and charge at any time. An example numerical configuration (a "snapshot" at one instant of time) is shown in Figure 4.1.

Regions I and III, semi-infinite in the PDE (2.12), are of finite length in the numerical scheme. In Region I, which stretches from $X=-L$ to $X=0$, there is a prescribed input signal; the update employed at the left-most extremity for producing it is derived in Appendix B.3. Fourier transforms of numerical time series at points in the regions I and III are used to evaluate the transmitted and reflected signals. Sinusoidal input signals may be accompanied by noise, either white or with constant power in a window of frequencies. The latter "band-limited" noise is generated either by filtering white noise or by explicitly constructing a signal as a sum of Fourier components with constant power in the frequency window.

Our analytical and numerical methods are not altered by changes in the form of the function $C(V)$, assuming that it is a positive even function with maximum at 0 . In the numerical runs producing the results displayed in Figures 4.1 to 4.5, we used the following form:

$$
C_{\mathrm{m}}(v)=C_{\mathrm{II}}\left(1+\left(\frac{v}{v^{*}}\right)^{2}\right)^{-1}
$$

giving the convenient explicit form $V(x, t)=\tan (Q(x, t))$ in region II and

$$
G_{\mathrm{n}}(V)=\frac{1}{1+V^{2}}
$$

The parameter values used in the numerical runs reported in this Section were 


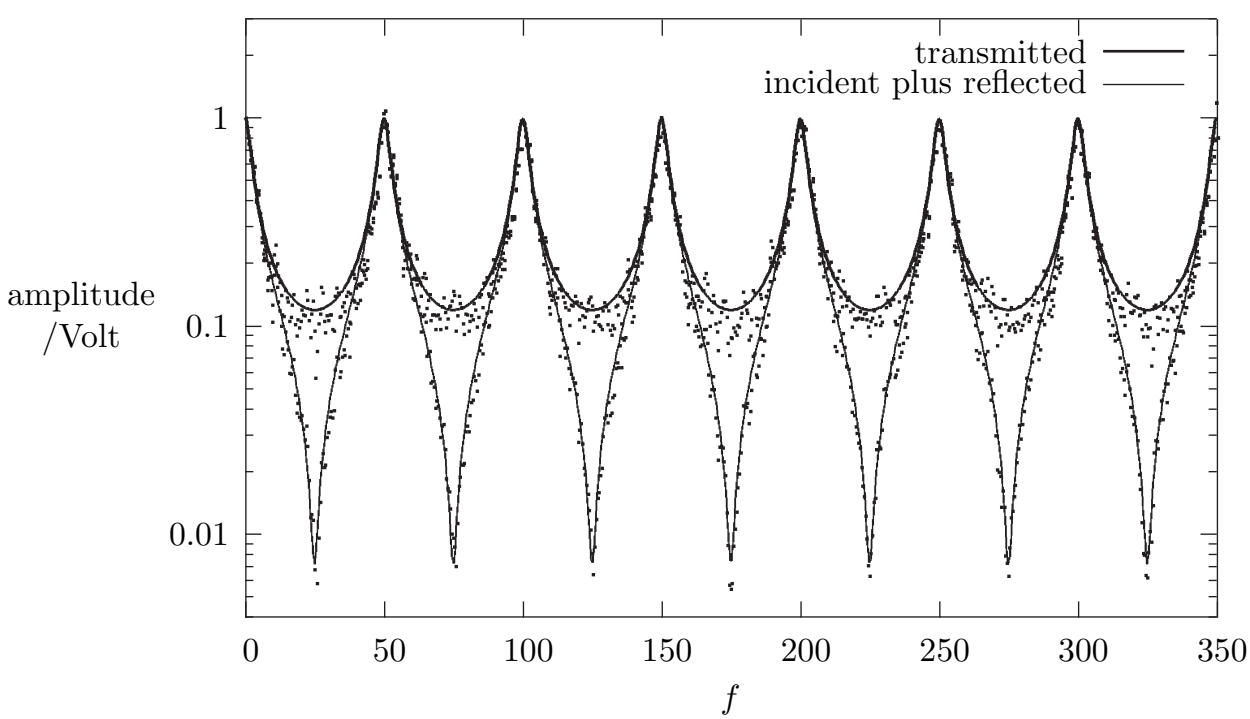

FIG. 4.2. The solid lines are modulus of the transmission coefficient $\mathcal{T}^{(0)}(\Omega)$ and of $1+\mathcal{R}^{(0)}(\Omega)$ as a function of frequency at (3.21). The dots are the Fourier transforms of numerical time series with white noise input.

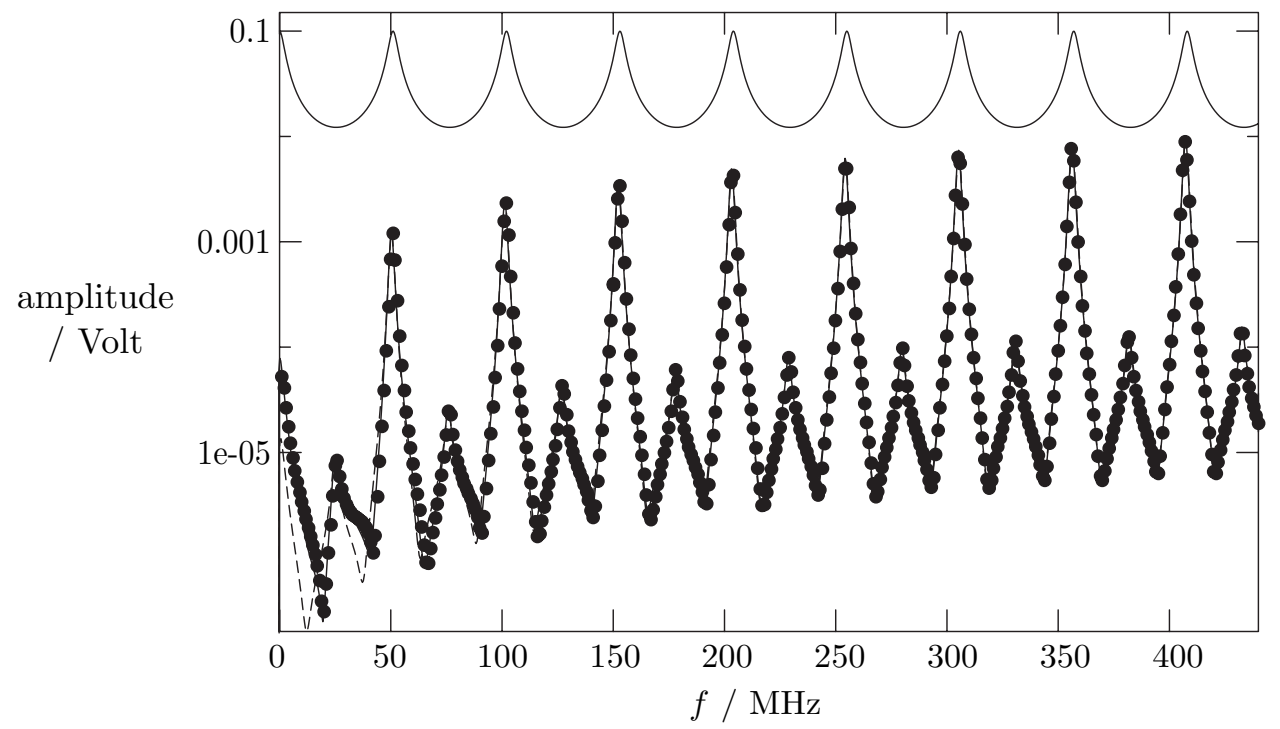

FIG. 4.3. Transmission and second-harmonic generation as a function of input frequency. The transmitted amplitudes at $f$ and $2 f$ are shown on a logarithmic scale. Numerical results are shown as solid circles and analytical results as lines. In particular, the dashed line corresponds to the high frequency limit (3.31), and, as seen, it becomes accurate past frequencies of about $50 \mathrm{MHz}$.

as follows. The length of Region II was taken to be $l=6 \mathrm{~cm}$ and the characteristic voltage $v^{*}=10 \mathrm{~V}$. The $L$ and $C$ constants in the Regions were given by $L_{\mathrm{I}} C_{\mathrm{I}} c^{2}=9$, $L_{\mathrm{II}} C_{\mathrm{II}} c^{2}=2500$. Thus the speed of light in regions I and III was one third of that in a vacuum, $c$. The corresponding speed in region II and the factor $\beta$ are bias-dependent; at zero bias the speed was $c / 50$ and $\beta=0.0036$. 

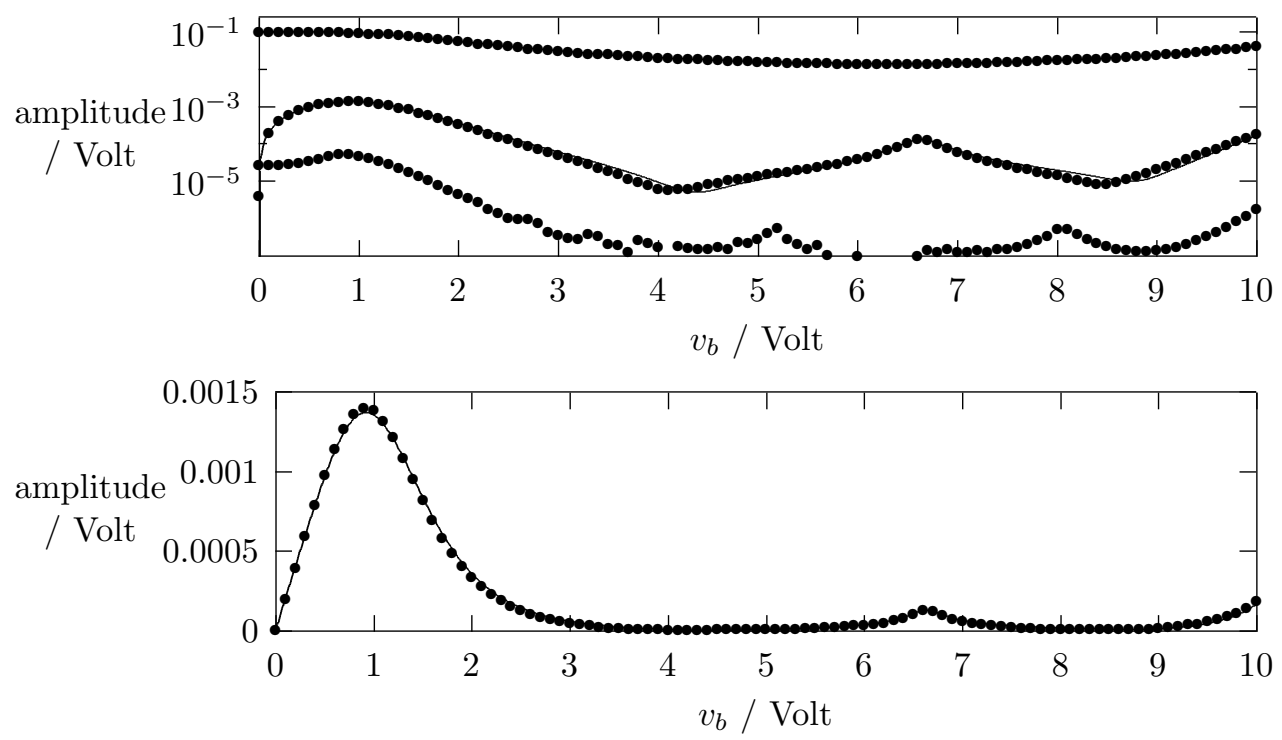

FIG. 4.4. Transmission and harmonic generation as a function of bias voltage. In the upper graph the output amplitudes at $f, 2 f$ and $3 f$ are shown on a logarithmic scale. In the lower graph the amplitude at $2 f$ is shown on a linear scale. Numerical results are shown as solid circles. The solid lines use (3.21) and (3.26). The dotted line, using (3.31), differs visibly from (3.26) only at low frequencies. In each case $f=150 \mathrm{MHz}$ and $a=0.1 \mathrm{~V}$.

The results of one numerical experiment are shown in Figure 4.2. The input was broad-band white noise with small amplitude, so that all frequencies have equal average amplitude in the input. The solid lines are the predictions obtained from (3.21). Assuming a flat input spectrum, we obtain the transmission coefficient $\mathcal{T}^{(0)}(\Omega)$ as the Fourier transform of the time series in region III, showing resonances when $\Omega / U_{m}$ was a multiple of $\pi$. (With $l=6 \mathrm{~cm}$, resonant frequencies are at multiples of $50 \mathrm{MHz}$.) The numerical results, shown as dots, fluctuate about the theoretical line as expected. In region I, the time-dependent voltage is the sum of incident and reflected signals; the Fourier amplitude in this case, shown in the figure, is $1+$ $\mathcal{R}^{(0)}(\Omega)$. At resonant frequencies $(3.22)$, there is perfect transmission and $\mathcal{R}^{(0)}(\Omega)=$ 0 . Halfway between resonant frequencies, at frequencies satisfying $\sin \left(\frac{\Omega}{U_{\mathrm{m}}}\right)= \pm 1$, we find minimum transmission

$$
\left|\mathcal{T}^{(0)}(\Omega)\right|_{\min }^{2}=\frac{4 \beta}{(1+\beta)^{2}}
$$

and

$$
\mathcal{R}^{(0)}(\Omega)=-\frac{1-\beta}{1+\beta}=-1+2 \beta+\mathcal{O}\left(\beta^{2}\right)
$$

at these frequencies $\mathcal{R}^{(0)}(\Omega) \simeq-1$, the reflected and incident signals almost cancel each other out. This feature is clearly visible in the numerical results. No nonlinear effects are displayed in Figure 4.2, but the run serves as a stringent check on the numerical algorithm over several orders of magnitude in voltage.

The effect of the nonlinearity in generating an output at twice the input frequency is shown in Figure 4.3, which summarizes the results of a series of numerical runs, 
each with a single sinusoidal input. The input amplitude $a=0.1 \mathrm{~V}$ was fixed and all runs were performed with bias voltage $v_{\mathrm{b}}=2.01$; the frequency $f$ of the input signal was changed from run to run. At the chosen bias voltage, resonant frequencies are multiples of $51 \mathrm{MHz}$. The Figure shows the transmitted amplitude at frequencies $f$ and $2 f$, along with the analytical predictions. The amplitude at $f$ is given by (3.21). The prediction at $2 f$, obtained by inverting (3.26), is remarkably accurate over many orders of magnitude in the transmitted amplitude. The asymptotic formula for high frequency (3.31), shown in this figure as a dashed line, is indistinguishable from the full inversion of (3.26) and the numerical data past frequencies of about $50 \mathrm{MHz}$.

The features of the second-harmonic amplitude predicted by (3.31) are clearly evident in the numerical results. The amplitude of the second harmonic is maximized when the frequency of the input signal is at resonance. Local maxima of the second harmonic amplitude are also found at frequencies halfway between resonances of the input signal. The height of the maxima coinciding with the $n$th resonance is proportional to $n$ because the second-harmonic amplitude is proportional to $\Omega$.

A similar series of runs was used to produce Figure 4.4, this time with all parameters except bias voltage held fixed. The input frequency is chosen to be at resonance at zero bias. The top graph shows the transmitted amplitude at $f, 2 f$ and $3 f$ on a logarithmic scale. (We have not attempted to calculate the $3 f$ amplitude, but conjecture that is is proportional to $C^{\prime \prime}\left(V_{\mathrm{b}}\right)$, and hence nonzero at $V_{\mathrm{b}}=0$.) In the lower Figure we plot the transmitted second-harmonic $(2 f)$ amplitude and the approximation (3.31) on a linear scale. At zero bias voltage, it is zero at order $A^{2}$ because $C^{\prime}(0)=0$. The second-harmonic amplitude at first increases with bias voltage, then decreases as the bias voltage is further increased, as the resonant frequency is shifted away from the input frequency. For sufficiently large bias voltage, the shift in resonant frequencies is such that the input frequency can once again be at resonance.

The convenient analytical forms (3.31), for the amplitudes and phases of the transmitted and reflected second harmonic at order $A^{2}$, give several intriguing possibilities if the input signal consists of two or more sinusoids. For example, in addition to a sinusoidal input at frequency $f$, another at frequency $2 f$ can be chosen so that the total transmission at $2 f$ is zero to order $A^{2}$, by cancellation of the second harmonic produced by the nonlinearity with the transmitted signal at $2 f$. This cancellation can be produced for any input frequency and will be explored further below in the case where $f$ is a resonant frequency. Alternatively, for an input frequency slightly off resonance, it is possible to choose a combination such that the reflected signal is free of harmonics to order $A^{2}$.

In Figure 4.5 we show the transmitted amplitude at $f$ and $2 f$, as a function of the phase $\phi$, when the input signal is given by

$$
v_{\text {in }}=a \cos (2 \pi f t)+b \cos (4 \pi f t+\phi),
$$

where $f$ is chosen to be the lowest resonant frequency at a non-zero bias voltage. At lowest order, the transmitted signal is equal to the input signal, since $2 f$ is also a resonant frequency. There is another source of transmitted power at $2 f$ : the generated second-harmonic signal with amplitude, given by (3.28), proportional to $a^{2} / v^{*}$. We choose $b$ in (4.5) to be equal to that amplitude. (With the parameters in Figure 4.5, $\left|\mathcal{T}^{(1)}(\Omega)\right| \simeq 1.3$ so $b=\left|\mathcal{T}^{(1)}(\Omega)\right| a^{2} / v^{*} \simeq 0.000013$.) According to (3.28), the phase of the generated $2 f$ signal is $\frac{1}{2} \pi$. Thus when $\phi=-\frac{1}{2} \pi$, the generated and directly transmitted outputs have equal amplitude and opposite phase; the result is to eliminate the total transmitted $2 f$ signal to order $A^{2}$. (Also shown in Figure 4.5, as a dotted line, 


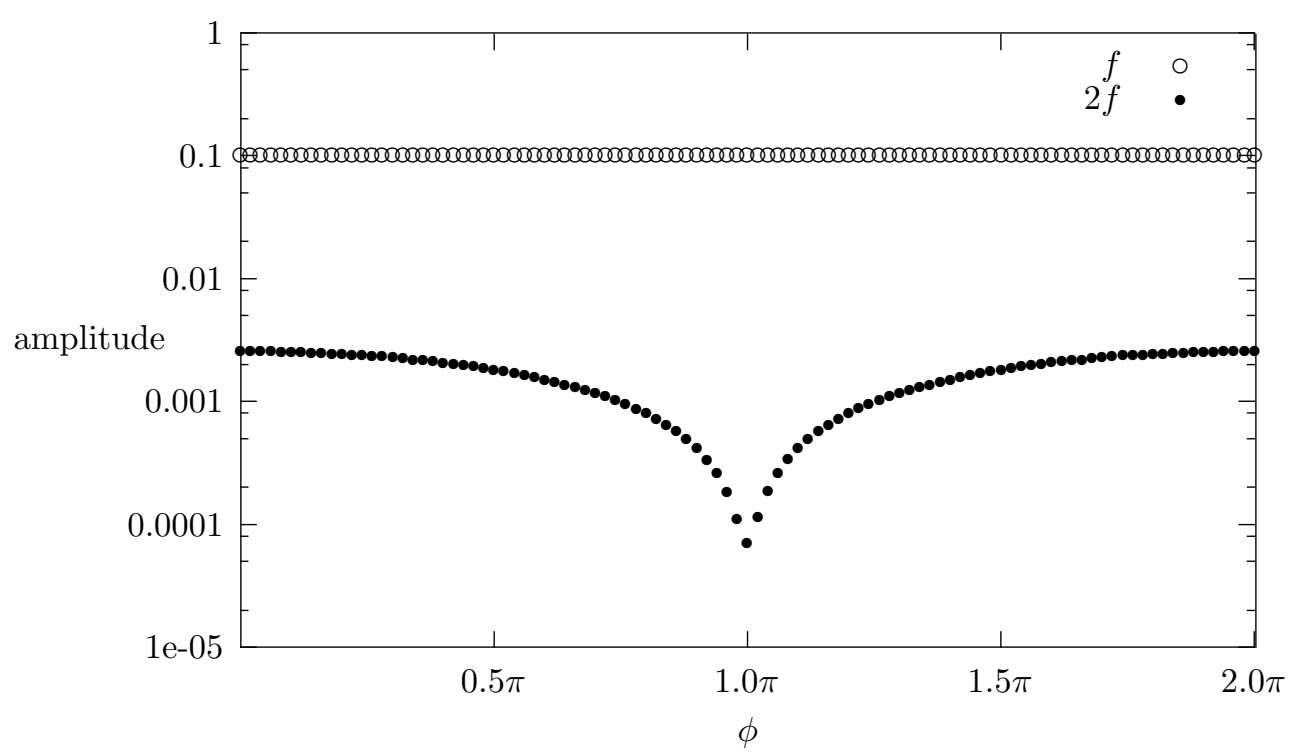

FIG. 4.5. Transmitted amplitude at $f$ and $2 f$ versus phase $\phi$, logarithmic scale. The input signal is given by (4.5), with the amplitude b chosen to be equal to that of the the second harmonic generated by the nonlinearity from the input at frequency $f$ with amplitude $a$. The parameters were $v_{\mathrm{b}}=2.01 \mathrm{~V}, f=51 \mathrm{MHz}$ and $a=0.01$.

is the transmitted amplitude at $f$, which is independent of $\phi$ and indistinguishable from the input amplitude.)

The procedure of producing a pure transmitted signal with frequency $f$ from a mixed input can in principle be carried to $n$th order, using an input signal with amplitudes and phases chosen to eliminate $n$ multiples of $f$ from the transmitted signal. The result would be a unique multi-frequency input that gives a pure single frequency transmitted signal through a given device. This uniqueness property, and its dependence on the external bias field, might have encoding implications in signal transmission protocols.

5. Comparison with an experimental system. In this section we report on experimental results. Microwave signals and noise were passed through a small device whose properties depend on operating temperature and applied voltage. A schematic diagram is shown in Figure 5.1. The devices are $1 \mathrm{~cm} \times 1 \mathrm{~cm} \times 0.5 \mathrm{~mm}$, consisting of $0.4 \mu$ m-thick superconducting $\mathrm{YBa}_{2} \mathrm{Cu}_{3} \mathrm{O}_{7}$ (YBCO) film on single-crystal substrates [22]. They are manufactured with two parallel meandering gaps (width $15 \mu \mathrm{m}$ ) patterned into the superconducting thin film. The result is a waveguide with a narrow meandering centerline (length $l \simeq 8 \mathrm{~cm}$ and width $20 \mu \mathrm{m}$ ) and two groundplanes (the rest of the superconducting film).

A constant voltage difference, the "bias" voltage, is maintained between the centerline and the groundplane. Because the lateral dimensions of the waveguide are much smaller than the wavelengths of the input signals, wave propagation is effectively one-dimensional. In the superconducting state, below the transition temperature $T_{\mathrm{c}} \simeq 85 \mathrm{~K}$, resistive losses of the YBCO film are negligible. Working temperatures were in the range $20 \mathrm{~K}-60 \mathrm{~K}$. The source of the nonlinearity in the experiment is the substrate that the superconducting waveguide rests on: a single crystal of strontium 


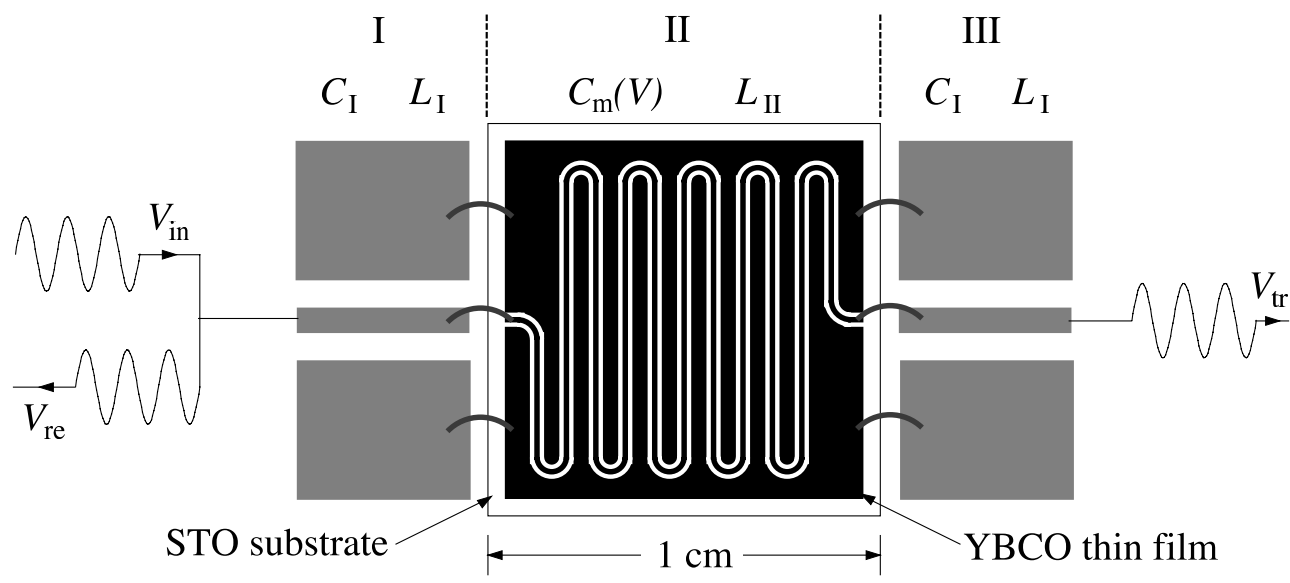

FIG. 5.1. Device top view and schematic diagram. The input circuitry, device, and output circuitry are modeled as regions I, II and III. The time-dependent voltage in region I is a sum of the incident and reflected sinusoidal signals, $V_{\text {in }}$ and $V_{\text {re. }}$ In the central region, region II, the wave equation is nonlinear. Signals travel along the meandering waveguide patterned into a superconducting thin film. The transmitted signal in region III, $V_{\mathrm{tr}}$, is measured and analyzed.

titanate, $\mathrm{SrTiO}_{3}$ (STO) with large permittivity. Due to the nonlinear dielectric properties of STO $[19,20]$, the shunt capacitance per unit length of the waveguide depends on temperature and on voltage. The resulting voltage-dependent differential capacitance produces behavior that combines resonance effects with harmonic generation and frequency mixing. More details can be found in [3], [10] and [11].

Our quantitative studies begin with a measurement of the differential capacitance as a function of temperature and voltage. We assume that the inductance per unit length, $L_{\mathrm{II}}$, is unaffected by changes in temperature and voltage. (The value $L_{\mathrm{II}}=505.7 \mathrm{pH} / \mathrm{mm}$ is deduced from measurements of the capacitance and resonant frequencies of the devices [11].) Measurement of the differential capacitance is conveniently carried out using a small-amplitude broadband noise input at various temperatures and bias voltages. The dominant feature of the transmitted spectrum is a series of maxima at resonant frequencies. The resonant frequencies at bias voltage $v_{\mathrm{b}}$ are $f_{n}\left(v_{\mathrm{b}}\right)$, where

$$
f_{n}\left(v_{\mathrm{b}}\right)=\frac{n}{2} \frac{u_{\mathrm{m}}\left(v_{\mathrm{b}}\right)}{l}, \quad n=0,1,2, \ldots,
$$

and the propagation speed for small-amplitude signals, $u_{\mathrm{m}}\left(v_{\mathrm{b}}\right)$, is given by $(2.14)$ : $u_{\mathrm{m}}\left(v_{\mathrm{b}}\right)=\left(L_{\mathrm{II}} C_{\mathrm{m}}\left(v_{\mathrm{b}}\right)\right)^{-\frac{1}{2}}$.

Experimental results for the transmitted signal as a function of input frequency are shown in Figure 5.2. The input in each case was broad-band white noise and the operating temperature was $40 \mathrm{~K}$. Because $C_{\mathrm{m}}\left(v_{\mathrm{b}}\right)$ is a decreasing function on $v_{\mathrm{b}}$, the distance between resonant peaks increases when a bias voltage is applied. The theoretical curve accurately gives the position of the resonant peaks in transmission, but does not predict their decreasing height as a function of frequency. The reason is that the model PDEs (2.12) do not include losses whose effect is to reduce transmission by a factor that increases with frequency.

The decreasing height of resonant peaks does not prevent us from accurately determining their position. Using (2.14) determines the differential capacitance as 

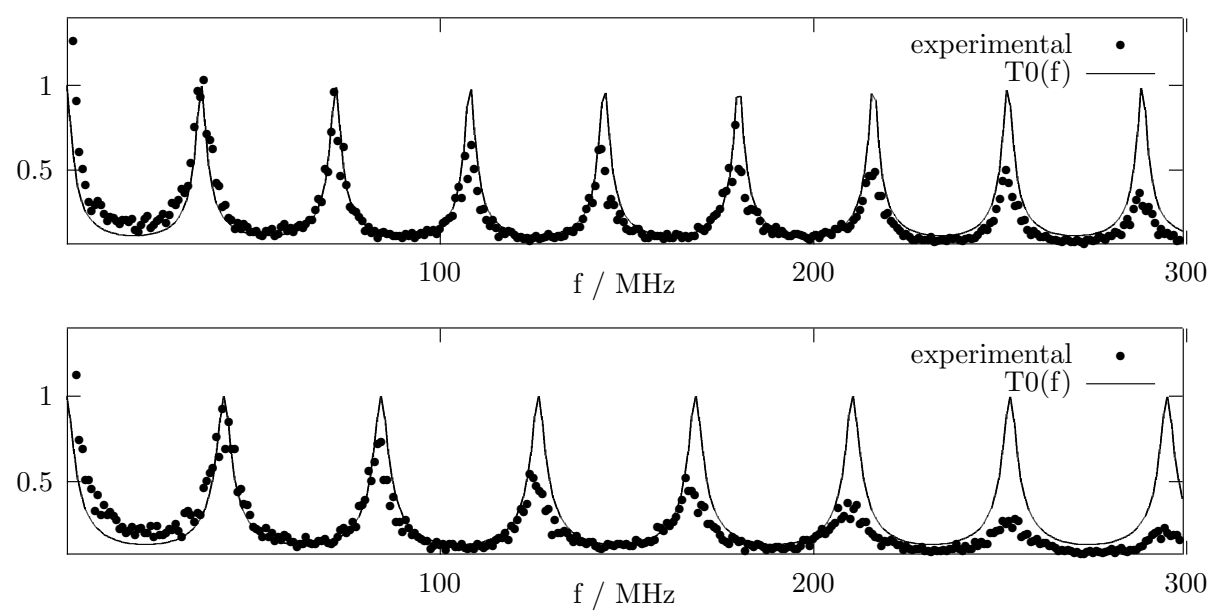

FIG. 5.2. Experimental and theoretical results: transmission vs frequency. The experimental results were obtained at operating temperature $40 \mathrm{~K}$. In the upper graph, bias voltage $=0$. In the lower graph, bias voltage $=10 \mathrm{~V}$. The solid lines are the theoretical curve (3.21), for linear lossless transmission. The parameters are $L_{\mathrm{I}} C_{\mathrm{I}} / L_{\mathrm{II}} C_{\mathrm{II}}=9 / 2500$ and $l=7.8 \mathrm{~cm}$. The positions of the resonance peaks are well predicted, but successive experimental peaks are of reduced height due to losses in the device.

a function of voltage; $C_{\mathrm{m}}\left(v_{\mathrm{b}}\right)$ is related to the frequency $f_{n}$, of the $n$th peak in the transmission-vs-frequency curve by

$$
L_{\mathrm{II}} C_{\mathrm{m}}\left(v_{\mathrm{b}}\right)=\left(\frac{n}{2} \frac{1}{l}\right)^{2} f_{n}^{-2}\left(v_{\mathrm{b}}\right)
$$

The upper plot in Figure 5.3 is the position of the third resonance as a function of bias voltage at operating temperature $40 \mathrm{~K}$. The function used to obtain the fit shown as a solid line is

$$
f\left(v_{\mathrm{b}}\right)=f(0)+c_{1} v_{\mathrm{b}} \tanh \left(c_{2} v_{\mathrm{b}}\right)
$$

where the two parameters $c_{1}$ and $c_{2}$ are adjusted once to obtain a fit through all the experimental points. The lower plot in Figure 5.3 is the ratio $C_{\mathrm{m}}\left(v_{\mathrm{b}}\right) / C_{\mathrm{II}}$, deduced from the upper curve using (5.2). In Figure 5.4, similar results are shown for operating temperature $20 \mathrm{~K}$. At this lower temperature, differential capacitance is a more rapidlyvarying function of bias voltage. As the figures show, the functional form (5.3) appears quite suitable for the purpose of modeling the medium's nonlinear response within the range of voltage amplitudes we have considered.

Figure 5.5 summarizes experimental data taken at $40 \mathrm{~K}$ using an input signal of fixed amplitude at $f=215 \mathrm{MHz}$. The frequency is chosen to be at the sixth resonance peak for zero bias voltage. The upper part of the plot shows the transmitted amplitude at $f$, normalized to its value at zero bias. As bias voltage increases away from zero, there is a shift in the resonance frequency (compare Figure 5.2) so that the input frequency is no longer a resonant frequency and the transmitted power decreases. The decrease is less rapid in the experiment (empty circles) than in the theoretical curve (dotted line) because losses have the effect of widening (as well as reducing the height of) resonant peaks. Dielectric losses are also known to decrease with bias 

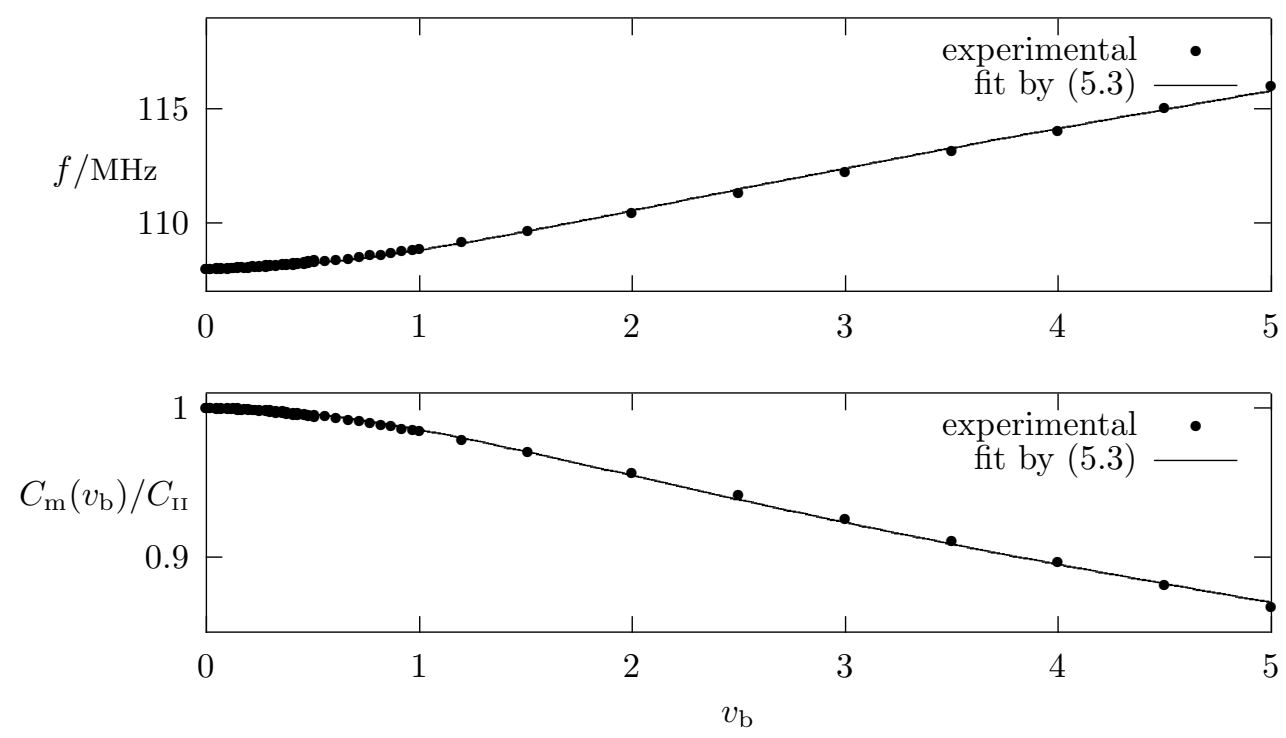

FIG. 5.3. Experimental results at operating temperature $40 \mathrm{~K}$ and best fit using (5.3). Upper graph: frequency at the $n=3$ peak as a function of bias voltage. Lower graph: Differential capacitance as a function of bias voltage divided by $C_{\mathrm{II}}=C_{\mathrm{m}}(0)$.
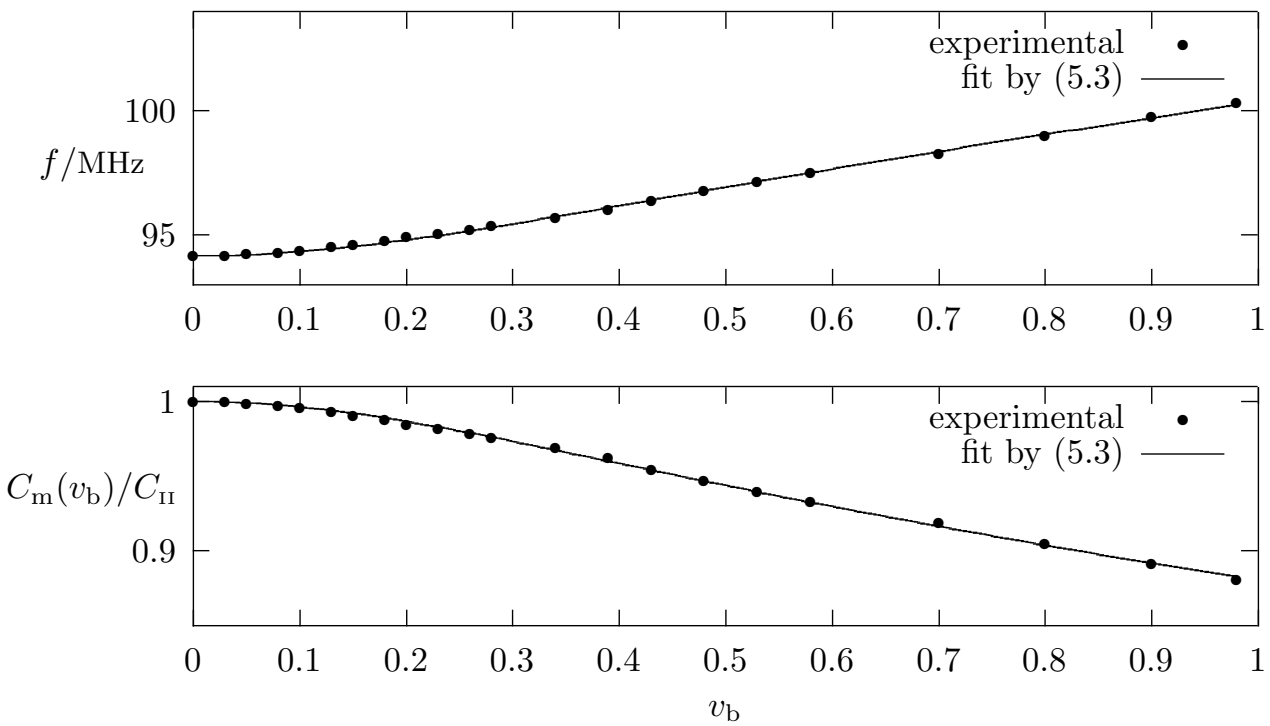

FIG. 5.4. Experimental results at operating temperature $20 K$ and best fit using (5.3). Upper graph: frequency at the $n=4$ peak as a function of bias voltage. Lower graph: Differential capacitance as a function of bias voltage divided by $C_{\mathrm{II}}=C_{\mathrm{m}}(0)$.

voltage $[22,24]$. The solid line and filled circles show the transmitted amplitude at $2 f$ as a function of bias. The reason for the initial rapid increase with bias is that the amplitude of the second harmonic signal is proportional to $C_{\mathrm{m}}^{\prime}\left(v_{\mathrm{b}}\right)$ and $C_{\mathrm{m}}^{\prime}(0)=0$. The subsequent decline is due to the decrease of the transmitted power at frequency $f$ (compare (3.31)). Also shown is the third harmonic power, which has a nonzero 


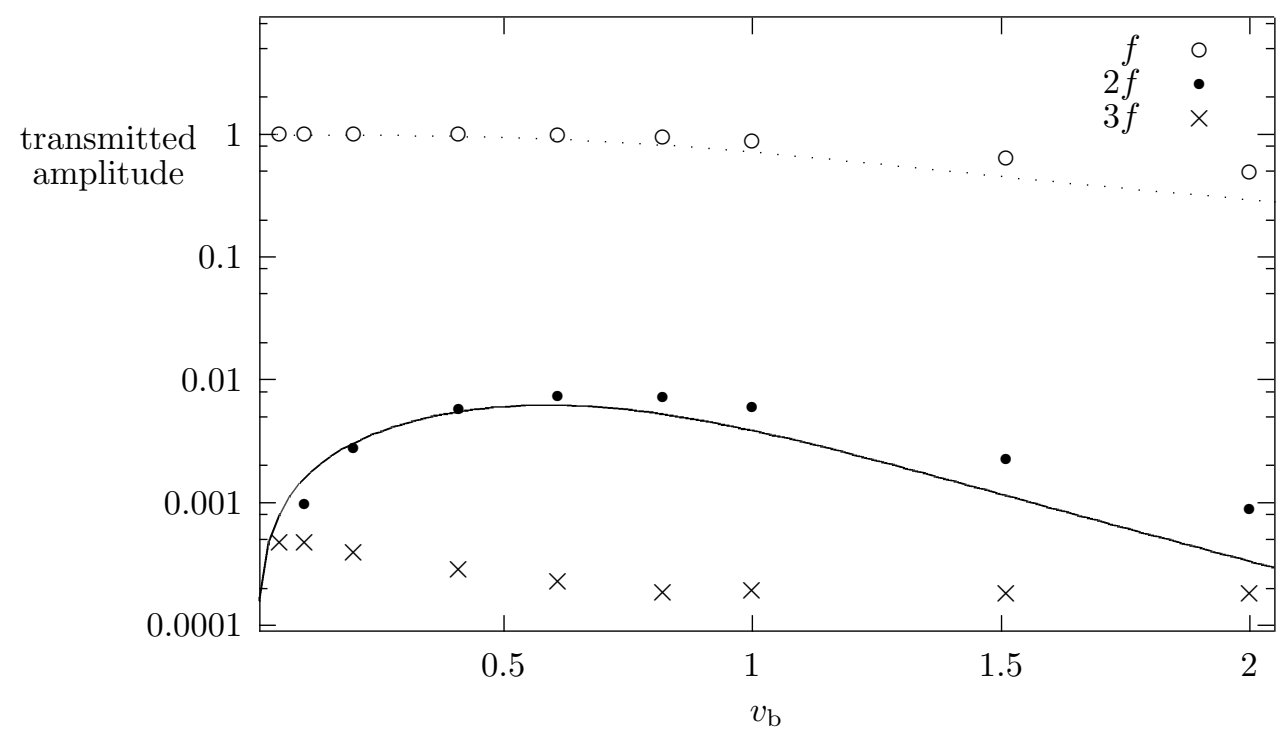

FIG. 5.5. Experimental results and theoretical curves: transmitted amplitudes at $f$ (empty circles), $2 f$ (filled circles) and $3 f$ (crosses) versus bias. The input is of fixed amplitude and frequency $f=215 \mathrm{MHz}$. The lines are the theoretical curves using asymptotic analysis of the lossless nonlinear $P D E$ (2.12). The dotted line is the transmission coefficient from the linear equation. The solid line shows the order $A^{2}$ second harmonic amplitude (3.31).

value at zero bias voltage and then decreases slowly [11].

6. Conclusion. A few points emerge from our side-by-side modeling and experimental investigation of electromagnetic propagation in a class of nonlinear waveguides. First, our simple voltage-current model seems to capture most of the physical features of the experimental device, including the tunable nonlinearity via an externally applied electric field. We find quantitative agreement with most measurable quantities. Some effects we neglect, such as dissipation, do show up in the data, but at least for the experimental regimes we have focused on they can be considered secondary.

Second, transmission of sinusoidal signals through a linear medium is maximized when the length of the region is equal to a multiple of half the wavelength. This remains the dominant feature in transmission through the nonlinear region we studied, because the signal amplitude is small enough and the nonlinear region is short enough that nonlinear and dispersive effects have a subdominant role. The main effect of the nonlinearity is to produce a second-harmonic output, with amplitude that is well predicted by an expansion that assumes that the input signal is sufficiently weak. The explicit and, especially near resonance, relatively simple formulae we have derived using an asymptotic approach perform well against both numerical solutions of our model equation and experimental results, thus giving us confidence that the theoretical building blocks we have established can be used in other studies of this class of materials.

Third, the numerical simulations in which we mix the input signal with a second harmonic show that it is possible to achieve transparency (or total reflection) at second order in the input amplitude. In principle the expansion we have set up could be carried out to higher orders, thus eliminating all higher harmonics from 
the transmitted signal. Such transparency, and its dependence on the external bias field, might be exploited for technological applications, such as signal transmission protocols.

The main difference between our system and optical systems [25] is the absence of frequency dispersion. Future work will apply and extend the tools we have developed in this paper to study arrays of similar devices, where the longer effective length will permit nonlinear pulses of permanent form (or "solitons") because of the dispersive effects that such configurations generate. Distributed (or, spatially-extended) nonlinear arrays have intrinsic broadband capability (at least several hundred $\mathrm{GHz}$ ) in contrast to electronic circuits based on discrete elements, and high-dimensional complexity, governed by PDEs or coupled ODEs, in contrast to low dimensional systems whose dynamics are governed by ODEs. Thus, distributed nonlinear arrays have the potential to handle wider data bandwidth at a high level of security in communication.

Acknowledgments. RC acknowledges support from NSF through Grant DMS0104329, and from DOE CCPP and BES programs through the Theoretical Division of Los Alamos National Laboratory. GL thanks Theoretical Division T7 and the Superconductivity Technology Center, Los Alamos National Laboratory, for hosting visits during which this work was completed.

\section{Appendix A. The initial times function.}

In this appendix we find the explicit lowest-order solution for the voltage in region II, evaluate the integral (3.25) that gives the initial times function and explicitly expand (3.7) to order $A^{2}$.

Consider a point $(T, X)$ in region II. It is the intersection of the two characteristics that intersect $X=0$ at $\left(T_{ \pm}(0), 0\right)$ where

$$
T_{ \pm}^{(0)}(0)=T \mp \frac{X}{U_{\mathrm{m}}} .
$$

Now the solution is constructed using

$$
V^{(0)}(X, T)=\left(2 G_{\mathrm{n}}\left(V_{\mathrm{b}}\right)^{\frac{1}{2}}\right)^{-1}\left(\Gamma_{+}^{(0)}(X, T)+\Gamma_{-}^{(0)}(X, T)\right) .
$$

Using the property of the characteristics, $\Gamma_{ \pm}(X, T)=\Gamma_{ \pm}\left(0, T_{ \pm}(0)\right)$, and the explicit forms,

$$
\begin{aligned}
V_{\mathrm{I}}^{(0)}(T) & =\frac{1}{2}\left(\left(1+\mathcal{R}^{(0)}(\Omega)\right) \mathrm{e}^{\mathrm{i} \Omega T}+\text { c.c. }\right), \\
I_{\mathrm{I}}^{(0)}(T) & =\left(\frac{L_{\mathrm{II}} C_{\mathrm{I}}}{L_{\mathrm{I}} C_{\mathrm{II}}}\right)^{\frac{1}{2}} \frac{1}{2}\left(\left(1-\mathcal{R}^{(0)}(\Omega)\right) \mathrm{e}^{\mathrm{i} \Omega T}+\text { c.c. }\right), \\
V_{\mathrm{III}}^{(0)}(T) & =\frac{1}{2}\left(\mathcal{T}^{(0)}(\Omega) \mathrm{e}^{\mathrm{i} \Omega T}+\text { c.c. }\right), \\
I_{\mathrm{III}}^{(0)}(T) & =\left(\frac{L_{\mathrm{II}} C_{\mathrm{I}}}{L_{\mathrm{I}} C_{\mathrm{II}}}\right)^{\frac{1}{2}} \frac{1}{2}\left(\mathcal{T}^{(0)}(\Omega) \mathrm{e}^{\mathrm{i} \Omega T}+\text { c.c. }\right),
\end{aligned}
$$

we obtain

$$
V^{(0)}(X, T)=G_{\mathrm{n}}\left(V_{\mathrm{b}}\right)^{-\frac{1}{2}} \frac{1}{2}\left[G_{\mathrm{n}}\left(V_{\mathrm{b}}\right)^{\frac{1}{2}} V_{\mathrm{I}}^{(0)}\left(T-\frac{X}{U_{\mathrm{m}}}\right)+I_{\mathrm{I}}^{(0)}\left(T-\frac{X}{U_{\mathrm{m}}}\right)\right.
$$




$$
\begin{gathered}
\left.+G_{\mathrm{n}}\left(V_{\mathrm{b}}\right)^{\frac{1}{2}} V_{\mathrm{I}}^{(0)}\left(T+\frac{X}{U_{\mathrm{m}}}\right)-I_{\mathrm{I}}^{(0)}\left(T+\frac{X}{U_{\mathrm{m}}}\right)\right] \\
=\frac{1}{2}\left[\exp \left(\mathrm{i} \Omega\left(T-\frac{X}{U_{\mathrm{m}}}\right)\right)+\mathcal{R}^{(0)}(\Omega) \exp \left(\mathrm{i} \Omega\left(T-\frac{X}{U_{\mathrm{m}}}\right)\right)\right. \\
+\beta^{\frac{1}{2}}\left(\exp \left(\mathrm{i} \Omega\left(T-\frac{X}{U_{\mathrm{m}}}\right)\right)-\mathcal{R}^{(0)}(\Omega) \exp \left(\mathrm{i} \Omega\left(T-\frac{X}{U_{\mathrm{m}}}\right)\right)\right) \\
+\exp \left(\mathrm{i} \Omega\left(T+\frac{X}{U_{\mathrm{m}}}\right)\right)+\mathcal{R}^{(0)}(\Omega) \exp \left(\mathrm{i} \Omega\left(T+\frac{X}{U_{\mathrm{m}}}\right)\right) \\
-\beta^{\frac{1}{2}}\left(\exp \left(\mathrm{i} \Omega\left(T+\frac{X}{U_{\mathrm{m}}}\right)\right)-\mathcal{R}^{(0)}(\Omega) \exp \left(\mathrm{i} \Omega\left(T+\frac{X}{U_{\mathrm{m}}}\right)\right)+\text { c.c. }\right] . \\
=\frac{1}{2}\left[\mathrm{e}^{\mathrm{i} \Omega T}\left(\left(1+\mathcal{R}^{(0)}(\Omega)\right) \cos \left(\frac{\Omega}{U_{\mathrm{m}}} X\right)-\mathrm{i} \beta^{\frac{1}{2}}\left(1-\mathcal{R}^{(0)}(\Omega)\right) \sin \left(\frac{\Omega}{U_{\mathrm{m}}} X\right)\right)+\text { c.c. }\right] .
\end{gathered}
$$

Thus

$$
\begin{aligned}
V^{(0)}(X, T)= & \left|1+\mathcal{R}^{(0)}(\Omega)\right| \cos \left(\frac{\Omega}{U_{\mathrm{m}}} X\right) \cos \left(\Omega T+\phi_{1}\right) \\
& -\beta^{\frac{1}{2}}\left|1-\mathcal{R}^{(0)}(\Omega)\right| \sin \left(\frac{\Omega}{U_{\mathrm{m}}} X\right) \sin \left(\Omega T+\phi_{2}\right),
\end{aligned}
$$

where $\phi_{1}=\arg \left(1+\mathcal{R}^{(0)}(\Omega)\right)$ and $\phi_{2}=\arg \left(1-\mathcal{R}^{(0)}(\Omega)\right)$. In the case of resonance, $\Omega=U_{\mathrm{m}} n \pi, \mathcal{R}^{(0)}(\Omega)=0$ and the solution in region II is simply

$$
V(X, T)=V_{\mathrm{b}}+A\left(\cos (n \pi X) \cos (\Omega T)-\beta^{\frac{1}{2}} \sin (n \pi X) \sin (\Omega T)\right) .
$$

To evaluate the integral in $(3.25)$,we need the quantity $V^{(0)}\left(X, T_{ \pm}^{(0)}(X)\right)$. Inserting

$$
T_{ \pm}^{(0)}(X)=S \mp \frac{1-X}{U_{\mathrm{m}}}
$$

and using (3.18) gives

$$
\begin{aligned}
& V^{(0)}\left(X, T_{ \pm}^{(0)}(X)\right)= \frac{1}{4}\left[\mathrm { e } ^ { \mathrm { i } \Omega ( S \mp U _ { \mathrm { m } } ^ { - 1 } ) } \left(1+\mathcal{R}^{(0)}(\Omega) \pm \beta^{\frac{1}{2}}\left(1-\mathcal{R}^{(0)}(\Omega)\right)+\right.\right. \\
&\left.\left.\quad\left(\cos \left(2 \frac{\Omega}{U_{\mathrm{m}}} X\right) \pm \mathrm{i} \sin \left(2 \frac{\Omega}{U_{\mathrm{m}}} X\right)\right)\left(1+\mathcal{R}^{(0)}(\Omega) \mp \beta^{\frac{1}{2}}\left(1-\mathcal{R}^{(0)}(\Omega)\right)\right)\right)+ \text { c.c. }\right] \\
&=\frac{1}{4}\left[\mathrm{e}^{\mathrm{i} \Omega S} \mathcal{T}^{(0)}(\Omega)\left(1 \pm \beta^{\frac{1}{2}}+\left(1 \mp \beta^{\frac{1}{2}}\right) \mathrm{e}^{\mp 2 \mathrm{i} \frac{\Omega}{U_{\mathrm{m}}}}\left(\cos \left(2 \frac{\Omega}{U_{\mathrm{m}}} X\right) \pm \mathrm{i} \sin \left(2 \frac{\Omega}{U_{\mathrm{m}}} X\right)\right)\right)+\text { c.c. }\right] .
\end{aligned}
$$

Thus, the next-to-lowest order term in the initial times function is explicitly

$$
\begin{aligned}
\tau_{ \pm}^{(1)}(S)=\mp U_{\mathrm{m}}^{-1} & \frac{1}{8} \frac{G_{\mathrm{n}}^{\prime}\left(V_{\mathrm{b}}\right)}{G_{\mathrm{n}}\left(V_{\mathrm{b}}\right)}\left[\mathrm { e } ^ { \mathrm { i } \Omega S } \mathcal { T } ^ { ( 0 ) } ( \Omega ) \left(1 \pm \beta^{\frac{1}{2}}\right.\right. \\
+ & \left.+\frac{1}{2} \frac{U_{\mathrm{m}}}{\Omega}\left(1 \mp \beta^{\frac{1}{2}}\right) \mathrm{e}^{\mp 2 \mathrm{i} \frac{\Omega}{U_{\mathrm{m}}}}\left(\sin \left(2 \frac{\Omega}{U_{\mathrm{m}}}\right) \pm \mathrm{i} \mp \mathrm{i} \cos \left(2 \frac{\Omega}{U_{\mathrm{m}}}\right)\right)+\text { c.c. }\right] .
\end{aligned}
$$


The full expression for (3.7), up to order $A^{2}$ with (3.14) and (3.17), is

$$
\begin{aligned}
& A G_{\mathrm{n}}\left(V_{\mathrm{b}}\right)^{\frac{1}{2}}\left(1 \pm \beta^{\frac{1}{2}}\right) \frac{1}{2}\left(\mathcal{T}^{(0)}(\Omega) \mathrm{e}^{\mathrm{i} \Omega S}+\text { c.c. }\right) \\
& +A^{2} G_{\mathrm{n}}\left(V_{\mathrm{b}}\right)^{\frac{1}{2}} V_{\mathrm{III}}^{(1)}(S) \pm A^{2} I_{\mathrm{III}}^{(1)}(S) \\
& +A^{2} G_{\mathrm{n}}\left(V_{\mathrm{b}}\right)^{\frac{1}{2}} \frac{1}{8} \frac{G_{\mathrm{n}}^{\prime}\left(V_{\mathrm{b}}\right)}{G_{\mathrm{n}}\left(V_{\mathrm{b}}\right)}\left(\left|\mathcal{T}^{(0)}(\Omega)\right|^{2}+\frac{1}{2}\left(\mathcal{T}^{(0)}(\Omega)^{2} \mathrm{e}^{2 \mathrm{i} \Omega S}+\text { c.c. }\right)\right) \\
& =A G_{\mathrm{n}}\left(V_{\mathrm{b}}\right)^{\frac{1}{2}} \frac{1}{2}\left[\mathrm{e}^{\mathrm{i} \Omega\left(S \mp U_{\mathrm{m}}^{-1}\right)}\left(1+\mathcal{R}^{(0)}(\Omega) \pm \beta^{\frac{1}{2}}\left(1-\mathcal{R}^{(0)}(\Omega)\right)\right)+\text { c.c. }\right] \\
& +A^{2} G_{\mathrm{n}}\left(V_{\mathrm{b}}\right)^{\frac{1}{2}} V_{\mathrm{I}}^{(1)}\left(S \mp U_{\mathrm{m}}^{-1}\right) \pm A^{2} I_{\mathrm{I}}^{(1)}\left(S \mp U_{\mathrm{m}}^{-1}\right) \\
& +A^{2} G_{\mathrm{n}}\left(V_{\mathrm{b}}\right)^{\frac{1}{2}} \frac{1}{8} \frac{G_{\mathrm{n}}^{\prime}\left(V_{\mathrm{b}}\right)}{G_{\mathrm{n}}\left(V_{\mathrm{b}}\right)}\left(\left|1+\mathcal{R}^{(0)}(\Omega)\right|^{2}+\frac{1}{2}\left[\left(1+\mathcal{R}^{(0)}(\Omega)\right)^{2} \mathrm{e}^{2 \mathrm{i} \Omega\left(S \mp U_{\mathrm{m}}^{-1}\right)}+\text { c.c. }\right]\right) \\
& +A^{2} G_{\mathrm{n}}\left(V_{\mathrm{b}}\right)^{\frac{1}{2}} \frac{1}{2} \Omega\left[\mathrm{ie}^{\mathrm{i} \Omega\left(S \mp U_{\mathrm{m}}^{-1}\right)}\left(1+\mathcal{R}^{(0)}(\Omega) \pm \beta^{\frac{1}{2}}\left(1-\mathcal{R}^{(0)}(\Omega)\right)\right)+\text { c.c. }\right] \tau_{ \pm}^{(1)}(S) .
\end{aligned}
$$

To order $A$ we regain (3.18). With (A.7), we obtain the following expression for (3.7) at order $A^{2}$ :

$$
\begin{aligned}
V_{\mathrm{III}}^{(1)}(S) \pm G_{\mathrm{n}}\left(V_{\mathrm{b}}\right)^{-\frac{1}{2}} I_{\mathrm{III}}^{(1)}(S)-V_{\mathrm{I}}^{(1)}\left(S \mp U_{\mathrm{m}}^{-1}\right) \mp G_{\mathrm{n}}\left(V_{\mathrm{b}}\right)^{-\frac{1}{2}} I_{\mathrm{I}}^{(1)}\left(S \mp U_{\mathrm{m}}^{-1}\right) \\
=\frac{1}{16} \frac{G_{\mathrm{n}}^{\prime}\left(V_{\mathrm{b}}\right)}{G_{\mathrm{n}}\left(V_{\mathrm{b}}\right)}\left[\left(-\left|\mathcal{T}^{(0)}(\Omega)\right|^{2}-\mathcal{T}^{(0)}(\Omega)^{2} \mathrm{e}^{2 \mathrm{i} \Omega S}+\left|\mathcal{T}^{(0)}(\Omega)\right|^{2}\left(\cos ^{2} \frac{\Omega}{U_{\mathrm{m}}}+\beta \sin ^{2} \frac{\Omega}{U_{\mathrm{m}}}\right)\right.\right. \\
\left.+\mathcal{T}^{(0)}(\Omega)^{2}\left(\cos ^{2} \frac{\Omega}{U_{\mathrm{m}}}-\mathrm{i} \beta^{\frac{1}{2}} \sin ^{2} \frac{\Omega}{U_{\mathrm{m}}}\right)^{2} \mathrm{e}^{2 \mathrm{i} \Omega\left(S \mp U_{\mathrm{m}}^{-1}\right)}+\text { c.c }\right) \\
\mp \frac{\Omega}{U_{\mathrm{m}}}\left(1 \pm \beta^{\frac{1}{2}}\right)\left(\left(1 \pm \beta^{\frac{1}{2}}+\frac{1}{2} \frac{U_{\mathrm{m}}}{\Omega}\left(\sin \left(2 \frac{\Omega}{U_{\mathrm{m}}}\right) \pm \mathrm{i}\left(1-\cos \left(2 \frac{\Omega}{U_{\mathrm{m}}}\right)\right)\right) \mathrm{i} \mathcal{T}^{(0)}(\Omega)^{2} \mathrm{e}^{2 \mathrm{i} \Omega S}+\text { c.c. }\right)\right] .
\end{aligned}
$$

\section{Appendix B. Numerical techniques.}

B.1. Lax-Wendroff finite-difference method. The fields $Q$ and $I$ are updated on a grid with spacing $\Delta X$. In the first-order Lax-Wendroff method, the values at $T+\Delta T$ are obtained from those at $T$ using [18]:

$$
\begin{aligned}
Q(X, T+\Delta T)= & \frac{1}{2}(Q(X+\Delta X, T)+Q(X, T)) \\
& -\frac{1}{2} U \frac{\Delta T}{\Delta X}(I(X+\Delta X, T)-I(X-\Delta X, T)), \\
I(X, T+\Delta T)= & \frac{1}{2}(I(X+\Delta X, T)+I(X, T)) \\
& -\frac{1}{2} U \frac{\Delta T}{\Delta X}(\mathcal{V}(Q(X+\Delta X, T))-\mathcal{V}(Q(X-\Delta X, T))) .
\end{aligned}
$$

We use the following second-order adaptation [18]:

$$
Q(X, T+\Delta T)=Q(X, T)-\frac{1}{2} U \frac{\Delta T}{\Delta X}(I(X+\Delta X, T)-I(X-\Delta X, T))
$$




$$
\begin{aligned}
+ & \frac{1}{2}\left(U \frac{\Delta T}{\Delta X}\right)^{2}(\mathcal{V}(Q(X+\Delta X, T))+\mathcal{V}(Q(X-\Delta X, T))) \\
I(X, T+\Delta T)= & I(X, T)-U \frac{\Delta T}{\Delta X}\left[\mathcal { V } \left(\frac{1}{2}(Q(X+\Delta X, T)+Q(X, T))\right.\right. \\
& \left.\quad-\frac{1}{2} U \frac{\Delta T}{\Delta X}(I(X+\Delta X, T)-I(X, T))\right) \\
- & \mathcal{V}\left(\frac{1}{2}(Q(X, T)+Q(X-\Delta X, T))\right. \\
& \left.\left.-\frac{1}{2} U \frac{\Delta T}{\Delta X}(I(X, T)-I(X-\Delta X, T))\right)\right]
\end{aligned}
$$

B.2. Discretized boundary conditions. At the interface between different media it is important to implement numerical boundary conditions that are of the same order of the scheme and do not produce artificial reflections[26]. Consider the boundary between regions I and II. Let $Q_{1}(n \Delta X, T)$ and $I_{1}(n \Delta X, T)$ be the values of the last point in region I; let $Q_{2}(0, T)$ and $I_{2}(0, T)$ be the values of the first point in region II. We cannot immediately implement the Lax-Wendroff scheme because the neighboring points to the boundary points are in different regions, where the equation of motion takes different forms: $\mathcal{V}(Q)=V_{1}(Q)$ and $\mathcal{V}(Q)=V_{\mathrm{n}}(Q)$.

Because $Q_{1}(n \Delta x, T)$ and $Q_{2}(0, T)$ correspond to the same position,

$$
I_{1}(n \Delta x, T)=I_{2}(0, T) \quad \text { and } \quad V_{1}\left(Q_{1}(n \Delta x, T)\right)=V_{\mathrm{n}}\left(Q_{2}(0, T)\right),
$$

with $V_{1}$ and $V_{\mathrm{n}}$ as defined in (2.11). As (B.5) is true for any $T$, we also have the equalities

$$
\frac{\partial}{\partial T} I_{1}(n \Delta x, T)=\frac{\partial}{\partial T} I_{2}(0, T)
$$

and

$$
\frac{\partial}{\partial T} V_{1}\left(Q_{1}(n \Delta x, T)\right)=\frac{\partial}{\partial T} V_{\mathrm{n}}\left(Q_{2}(0, T)\right)
$$

Therefore, from the equation of motion (2.12),

$$
\frac{\partial}{\partial X} V_{1}\left(Q_{1}(n \Delta x, T)\right)=\frac{\partial}{\partial X} V_{\mathrm{n}}\left(Q_{2}(0, T)\right)
$$

and

$$
V_{1}^{\prime}\left(Q_{1}(n \Delta x, T)\right) \frac{\partial}{\partial X} I_{1}(n \Delta x, T)=V_{\mathrm{n}}^{\prime}\left(Q_{2}(0, T)\right) \frac{\partial}{\partial X} I_{2}(0, T)
$$

where

$$
V_{1}^{\prime}(Q)=\frac{\partial}{\partial Q} V_{1}(Q) \quad V_{\mathrm{n}}^{\prime}(Q)=\frac{\partial}{\partial Q} V_{\mathrm{n}}(Q) .
$$

The discrete approximation to the partial derivatives on the right-hand side of (B.9) is

$$
\begin{aligned}
& \text { (B.11 } \frac{\partial}{\partial X} I_{1}(n \Delta x, T)=(2 \Delta x)^{-1}\left(3 I_{1}(n \Delta x)-4 I_{1}((n-1) \Delta x)+I_{1}((n-2) \Delta x)\right), \\
& \text { (B.12) } \frac{\partial}{\partial X} I_{2}(0, T)=(2 \Delta x)^{-1}\left(-3 I_{2}(0)+4 I_{2}(\Delta x)-I_{2}(2 \Delta x)\right)
\end{aligned}
$$


the derivatives in (B.8) are evaluated similarly. The discretized version of (B.8)-(B.9) is therefore

$$
\begin{aligned}
V_{\mathrm{l}}\left(Q_{1}(n \Delta x, T)\right)= & V_{\mathrm{n}}\left(Q_{2}(0, T)\right) \\
= & \frac{1}{6}\left(4 V_{\mathrm{l}}\left(Q_{1}((n-1) \Delta x, T)\right)-V_{1}\left(Q_{1}((n-2) \Delta x, T)\right)\right. \\
+ & \left.4 V_{\mathrm{n}}\left(Q_{2}(\Delta x, T)\right)-V_{\mathrm{n}}\left(Q_{2}(2 \Delta x, T)\right)\right), \\
I_{1}(n \Delta x, T)= & I_{2}(0, T) \\
= & \frac{1}{3}\left(1+\frac{V_{\mathrm{n}}^{\prime}\left(Q_{2}(0, T)\right)}{V_{1}^{\prime}\left(Q_{1}(n \Delta x, T)\right)}\right)^{-1}\left(4 I_{1}((n-1) \Delta x)-I_{1}((n-2) \Delta x)\right. \\
& \left.+\frac{V_{\mathrm{n}}^{\prime}\left(Q_{2}(0, T)\right)}{V_{1}^{\prime}\left(Q_{1}(n \Delta x, T)\right)}\left(4 I_{2}(\Delta x)-I_{2}(2 \Delta x)\right)\right) .
\end{aligned}
$$

B.3. Input signal. At the left-hand extremity of region I, $X=-L$, the solution of the field equation (2.12) can be written as a superposition of two fields: a prescribed incident wave $Q_{\text {in }}(X-c T), I_{\text {in }}(X-c T)$, and an (unknown) outgoing wave $Q_{\text {out }}(X+$ $c T), I_{\text {out }}(X+c T)(c>0)$. Thus, the incoming field satisfies the unidirectional wave equation

$$
\frac{\partial}{\partial T}\left(\begin{array}{c}
Q_{\text {in }} \\
I_{\text {in }}
\end{array}\right)=-c \frac{\partial}{\partial X}\left(\begin{array}{c}
Q_{\text {in }} \\
I_{\text {in }}
\end{array}\right)
$$

while the outgoing field satisfies

$$
\frac{\partial}{\partial T}\left(\begin{array}{c}
Q_{\text {out }} \\
I_{\text {out }}
\end{array}\right)=+c \frac{\partial}{\partial X}\left(\begin{array}{c}
Q_{\text {out }} \\
I_{\text {out }}
\end{array}\right)
$$

Hence, for the total field $Q(X, T), I(X, T)$,

$$
\begin{aligned}
\frac{\partial}{\partial T}\left(\begin{array}{c}
Q \\
I
\end{array}\right) & =\frac{\partial}{\partial T}\left(\begin{array}{c}
Q_{\text {in }} \\
I_{\text {in }}
\end{array}\right)+\frac{\partial}{\partial T}\left(\begin{array}{c}
Q_{\text {out }} \\
I_{\text {out }}
\end{array}\right) \\
& =\frac{\partial}{\partial T}\left(\begin{array}{c}
Q_{\text {in }} \\
I_{\text {in }}
\end{array}\right)+c \frac{\partial}{\partial X}\left(\begin{array}{c}
Q_{\text {out }} \\
I_{\text {out }}
\end{array}\right) \\
& =\frac{\partial}{\partial T}\left(\begin{array}{c}
Q_{\text {in }} \\
I_{\text {in }}
\end{array}\right)+c \frac{\partial}{\partial X}\left(\begin{array}{c}
Q-Q_{\text {in }} \\
I-I_{\text {in }}
\end{array}\right) .
\end{aligned}
$$

Using (B.13), the governing equation of the total field in the left linear region can then be rewritten as

$$
\frac{\partial}{\partial T}\left(\begin{array}{c}
Q \\
I
\end{array}\right)=2 \frac{\partial}{\partial T}\left(\begin{array}{c}
Q_{\text {in }} \\
I_{\text {in }}
\end{array}\right)+c \frac{\partial}{\partial X}\left(\begin{array}{c}
Q \\
I
\end{array}\right)
$$

this is used with one-sided spatial derivatives to construct the field at $X=-L$ from the knowledge of the field at the previous time step.

For example, consider a sinusoidal input signal, as in (3.1). Then, using (3.2),

$$
V_{\text {in }}(X, T)=A \cos \left(\Omega\left(T-X / U_{\mathrm{I}}\right)\right) .
$$


The incident $Q$ and $I$ fields are given by

$$
\begin{aligned}
Q_{\text {in }}(X, T) & =\frac{C_{\mathrm{I}}}{C_{\mathrm{II}}} A \cos \left(\Omega\left(T-\frac{X}{U_{\mathrm{I}}}\right)\right), \\
I_{\mathrm{in}}(X, T) & =\left(\frac{L_{\mathrm{II}} C_{\mathrm{I}}}{L_{\mathrm{I}} C_{\mathrm{II}}}\right)^{\frac{1}{2}} A \cos \left(\Omega\left(T-\frac{X}{U_{\mathrm{I}}}\right)\right) .
\end{aligned}
$$

Thus the (lowest-order) increments at the left extremity of region I are

$$
\begin{aligned}
Q_{1}(-L, T+\Delta T)= & Q_{1}(-L, T)+\Delta T\left(2 \frac{\mathrm{d}}{\mathrm{d} t} Q_{\text {in }}(-L, T)\right. \\
& \left.-U_{\mathrm{I}}(2 \Delta x)^{-1}\left(3 Q_{1}(0)-4 Q_{1}(-L+\Delta x)+Q_{1}(-L+2 \Delta x)\right)\right), \\
I_{1}(-L, T+\Delta T)= & I_{1}(-L, T)+\Delta T\left(2 \frac{\mathrm{d}}{\mathrm{d} t} I_{\mathrm{in}}(-L, T)\right. \\
& \left.-U_{\mathrm{I}}(2 \Delta x)^{-1}\left(3 I_{1}(0)-4 I_{1}(-L+\Delta x)+I_{1}(-L+2 \Delta x)\right)\right) .
\end{aligned}
$$

\section{REFERENCES}

[1] Robert E. Collins, Foundations for microwave engineering, McGraw-Hill, New York, 1992.

[2] V.K. Varadan, D.K. Ghodgaonkar, V.V. Varadan, J.F. Kelly and P. Glikerdas, Ceramic phase shifters for electronically steerable antenna systems, Microwave J. 35 (1992) p. 116.

[3] A. T. Findikoglu, Q. X. Jia, D. W. Reagor and X. D. Wu, Tunable Microwave Mixing in Nonlinear Dielectric Thin Films of $\mathrm{SrTiO}_{3}$ and $\mathrm{SrO}_{5} \mathrm{BaO}_{5} \mathrm{TiO}_{3}$ Electron. Lett. 31 (1995) pp. 1814-1815.

[4] F.W. VanKeuls, R.R. Romanofsky and D.Y. Bohman, M.D. Winters, F.A. Miranda, C.H. Mueller, R.E. Treece, T.V. Rivkin, D. Galt, $\mathrm{YBa}_{2} \mathrm{Cu}_{3} \mathrm{O}_{7}$-delta,Au)/SrTiO $3 / \mathrm{LaAlO}_{3}$ thin film conductor ferroelectric coupled microstripline phase shifters for phased array applications Appl. Phys. Lett. 71 (1997) pp. 3075-3077.

[5] G. Subramanyam, F. VanKeuls and F.A. Miranda, A K-band tunable microstrip bandpass filter using a thin-film conductor/ferroelectric/dielectric multilayer configuration, IEEE Microwave Guided Wave Lett. 8 (1998) pp. 78-80.

[6] A.B. Kozyrev et. Al., Nonlinear behavior of thin film $\mathrm{SrTiO}_{3}$ capacitors at microwave frequencies J. Appl. Phys. 84 (1998) pp. 3326-3332.

[7] H. Fuke, Y. Terashima, H. Kayano and H. Yoshino, Electrically tunable $\mathrm{YBa}_{2} \mathrm{Cu}_{3} \mathrm{O}_{y}$ resonators using interdigital electrodes and dielectric film, Physica C 336 (2000) pp. 80-84.

[8] G. Subramanyam, F.W. VanKeuls and F.A. Miranda, Effect of DC biasing on YBCO/STO/LAO tunable microstrip filters, Integ. Ferroelec. 29 (2000) pp. 81-93.

[9] G. Subramanyam, F.W. VanKeuls, F.A. Miranda, R.R. Romanofsky and J.D. Warner, Design and development of ferroelectric tunable HTS microstrip filters for $\mathrm{Ku}$ - and $\mathrm{K}$-band applications, Mat. Chem. Phys. 79 (2003) pp. 147-150.

[10] A. T. Findikoglu, Roberto Camassa, Grant Lythe and Q.X. Jia, New Potential Applications of Nonlinear Dielectrics: Microwave Solitons and Stochastic Resonance, Integ. Ferroelec. 22 pp. 259-268 (1998).

[11] Alp Findikoglu, Roberto Camassa, Grant Lythe and Q.X. Jia, Dielectric nonlinearity and stochastic effects in strontium titanate, Appl. Phys. Lett. 80 (2002) pp. 3391-3393.

[12] A. T. Findikoglu, Q. X. Jia, I.H. Campbell, X.D. Wu, D. W. Reagor, C.B. MombourQUETTE AND D. MuRRAY, Electrically tunable coplanar transmission line resonators using $\mathrm{YBa}_{2} \mathrm{Cu}_{3} \mathrm{O}_{7-x} / \mathrm{SrTiO}_{3}$ bilayers Appl. Phys. Lett. 66 (1995) pp. 3674-3676.

[13] M.J. Lancaster, J. Powell, A. Porch, Thin-film ferroelectric microwave devices, Superconductor Science and Technology 11 (1998) pp. 1323-1334.

[14] Q. X. Jia, A. T. Findikoglu, D. W. Reagor, P. Lu, Improvement in performance of electrically tunable devices based on nonlinear dielectric $\mathrm{SrTiO}_{3}$ using a homoepitaxial $\mathrm{LaAlO}_{3}$ interlayer, Appl. Phys. Lett. 73 (1998) pp. 897-899.

[15] A. T. Findikoglu, Q. X. Jia, X. D. Wu, and D. W. Reagor, Paraelectric Thin Films for Microwave Applications Integ. Ferroelec. 15 (1997) pp. 163-171.

[16] Phoolan Prasad and Renuka Ravindran Partial differential equations, Wiley Eastern, New Delhi, 1985. 
[17] Albert Messiah Quantum Mechanics, North-Holland, Amsterdam, 1962.

[18] W.H. Press, B.P. Flannery, S.A. Teukolsky and W.T. Vetterling Numerical Recipes the Art of Scientific Computing, Cambridge University Press, Cambridge, 1986.

[19] R.C. Neville, C.A. Mead And B. Hoeneise Permittivity of Strontium Titanate, J. Appl. Phys. 43 (1972) pp. 2124-2131.

[20] O.G. Vendik, Dielectric nonlinearity of the displacive ferroelectrics at UHF, Ferroelectrics 12 (1976) pp. 85-90.

[21] M.J. Dalberth, R.E. Stauber J.C. Price, C.T. Rogers and D. Galt, Improved low frequency and microwave dielectric response in strontium titanate thin films grown by pulsed laser ablation, Appl. Phys. Lett. 72 (1998) pp. 507-509.

[22] A.T. Findikoglu, D.W. Reagor , K.O. Rasmussen, A.R. Bishop, N. Gronbech-Jensen, Q.X. JiA, Y. FAN, C. KWON, L.A. OstrovsKY Electrodynamic properties of coplanar waveguides made from high-temperature superconducting YBa2Cu3O7? delta electrodes on nonlinear dielectric $\mathrm{SrTiO}_{3}$ substrates J. Appl. Phys. 86 (1999) pp. 1558-1568

[23] The FORTRAN program used to produce the numerical results discussed in this Section can be viewed at

http://www.maths.leeds.ac.uk/Applied/stochastic/scan10.f

and a sample input file at http://www.maths.leeds.ac.uk/Applied/stochastic/scan.in.

[24] O.G. Vendik, L.T. Ter-Martirosyan, S.P. ZuBK, Microwave losses in incipient ferroelectrics as functions of the temperature and the biasing field, Journal of Applied Physics 84 (1998) pp. 993-998.

[25] Paul Mandel Theoretical Problems in Cavity Nonlinear Optics, Cambridge University Press, Cambridge, 1997.

[26] J.M. Hyman, A method of lines approach to the numerical solutions of conservation laws, in Advances in Computer Methods for Partial Differential Equations III, R. Vichnevetsky and R.S. Stepleman eds., Publ. IMACS, Piscataway, 1978. 